\title{
Versal Deformations of Leibniz Algebras
}

\author{
Alice Fialowski $\stackrel{*}{\text { Ashis Mandal and Goutam Mukherjee* }}$
}

June 23, 2018

\begin{abstract}
In this work we consider deformations of Leibniz algebras over a field of characteristic zero. The main problem in deformation theory is to describe all non-equivalent deformations of a given object. We give a method to solve this problem completely, namely work out a construction of a versal deformation for a given Leibniz algebra, which induces all non-equivalent deformations and is unique on the infinitesimal level.
\end{abstract}

Keywords: Leibniz algebra, Leibniz cohomology, infinitesimal deformation, versal deformation, obstruction.

Mathematics Subject Classifications (2000): 13D10, 14D15, $13 \mathrm{D} 03$.

\section{Introduction}

Deformations of different algebraic and analytic objects are an important aspect if one studies their properties. They characterize the local behavior in a small neighborhood in the variety of a given type objects.

After the classical work of Gerstenhaber in the 60's [6, 7, 8, 9, 10, formal deformation theory was generalized in different categories. Computations were made, but the question of obtaining all non-equivalent deformations of a given object was not properly discussed for a long time. The right approach to this is the notion of versal deformation: a deformation which induces all non-equivalent ones. The existence of such a "versal" deformation for algebraic categories follows from the work of Schlessinger [22]. For Lie algebras it was worked out in [4], and one can deduce it to other categories as well. It turns out that (under some minor cohomology restrictions) there exists a versal element, which is universal at the infinitesimal level.

\footnotetext{
* The work was supported by grants from INSA, the Hungarian Academy of Sciences and by grants OTKA T043641, T043034.
} 
In this work we consider Leibniz algebras and give a construction for a versal element. It is parallel to the general constructions in deformation theory as in 14, 21, 16, 11, 15. The first specific method was given for Lie algebras in [5]. Here we are going to work out a similar construction for Leibniz algebras, suitable for explicit computations.

The structure of the paper is as follows: In Section 2 we give the necessary definitions for Leibniz algebras and their cohomology. In Section 3 we define the

notion of deformation over a commutative local algebra base and introduce some basic definitions related to deformations. In Section 4 we construct a specific infinitesimal deformation of a Leibniz algebra, which turns out to be universal in the class of infinitesimal deformations. We also introduce the notion of versal deformation: a deformation which is unique on the infinitesimal level and induces all other deformations. Section 5 deals with obstructions of extending a deformation, and in Section 6 we give the construction of a versal deformation.

\section{Leibniz Algebra and its Cohomology}

Leibniz algebras were introduced by J.L.-Loday [17, 19] and their cohomology was defined in 20,18$]$. Let us recall some basic definitions.

Definition 2.1. A Leibniz algebra is a $\mathbb{K}$-module $L$, where $\mathbb{K}$ is a field, equipped with a bracket operation that satisfies the Leibniz identity:

$$
[x,[y, z]]=[[x, y], z]-[[x, z], y], \text { for } x, y, z \in L .
$$

Any Lie algebra is automatically a Leibniz algebra, as in the presence of antisymmetry, the Jacobi identity reduces to the Leibniz identity. More examples of Leibniz algebras were given in [20, and recently for instance in [1, 2].

Let $L$ be a Leibniz algebra and $M$ a representation of $L$. By definition, $M$ is a $\mathbb{K}$-module equipped with two actions (left and right) of $L$,

$$
\begin{gathered}
{[-,-]: L \times M \longrightarrow M \text { and }[-,-]: M \times L \longrightarrow M \text { such that }} \\
{[x,[y, z]]=[[x, y], z]-[[x, z], y]}
\end{gathered}
$$

holds, whenever one of the variables is from $M$ and the two others from $L$.

Define $C L^{n}(L ; M):=\operatorname{Hom}_{\mathbb{K}}\left(L^{\otimes n}, M\right), n \geq 0$. Let

$$
\delta^{n}: C L^{n}(L ; M) \longrightarrow C L^{n+1}(L ; M)
$$


be a $\mathbb{K}$-homomorphism defined by

$$
\begin{aligned}
& \delta^{n} f\left(x_{1}, \cdots, x_{n+1}\right) \\
& :=\left[x_{1}, f\left(x_{2}, \cdots, x_{n+1}\right)\right]+\sum_{i=2}^{n+1}(-1)^{i}\left[f\left(x_{1}, \cdots, \hat{x}_{i}, \cdots, x_{n+1}\right), x_{i}\right] \\
& +\sum_{1 \leq i<j \leq n+1}(-1)^{j+1} f\left(x_{1}, \cdots, x_{i-1},\left[x_{i}, x_{j}\right], x_{i+1}, \cdots, \hat{x}_{j}, \cdots, x_{n+1}\right) .
\end{aligned}
$$

Then $\left(C L^{*}(L ; M), \delta\right)$ is a cochain complex, whose cohomology is called the cohomology of the Leibniz algebra $L$ with coefficients in the representation $M$. The $n$-th cohomology is denoted by $H L^{n}(L ; M)$. In particular, $L$ is a representation of itself with the obvious action given by the bracket in $L$. The $n$-th cohomology of $L$ with coefficients in itself is denoted by $H L^{n}(L ; L)$.

\section{Deformations}

We introduce the notion of deformation of a Leibniz algebra over a commutative algebra base. For an analogous definition for Lie algebras see [4, 5]. Fix a field $\mathbb{K}$ of characteristic zero. Let $L$ be a Leibniz algebra over $\mathbb{K}$ and $A$ a commutative algebra with identity over $\mathbb{K}$. Let $\varepsilon: A \rightarrow \mathbb{K}$ be a fixed augmentation, that is an algebra homomorphism with $\varepsilon(1)=1$ and $\operatorname{ker}(\varepsilon)=\mathfrak{M}$. Throughout the paper we shall assume that $\operatorname{dim}\left(\mathfrak{M}^{k} / \mathfrak{M}^{k+1}\right)<\infty$ for all $k$.

Definition 3.1. A deformation $\lambda$ of $L$ with base $(A, \mathfrak{M})$, or simply with base $A$, is an A-Leibniz algebra structure on the tensor product $A \otimes_{\mathbb{K}} L$ with the bracket $[,]_{\lambda}$ such that

$$
\varepsilon \otimes i d: A \otimes L \rightarrow \mathbb{K} \otimes L
$$

is a A-Leibniz algebra homomorphism (where the A-Leibniz algebra structure on $\mathbb{K} \otimes L$ is given via $\varepsilon$ ).

A deformation of the Leibniz algebra $L$ with base $A$ is called local if $A$ is a local algebra over $\mathbb{K}$, and is called infinitesimal (or first order) if, in addition to this, $\mathfrak{M}^{2}=0$. Observe that for $l_{1}, l_{2} \in L$ and $a, b \in A$ we have

$$
\left[a \otimes l_{1}, b \otimes l_{2}\right]_{\lambda}=a b\left[1 \otimes l_{1}, 1 \otimes l_{2}\right]_{\lambda}
$$

by $A$ - linearity of $[,]_{\lambda}$. Thus to define a deformation $\lambda$ it is enough to specify the brackets $\left[1 \otimes l_{1}, 1 \otimes l_{2}\right]_{\lambda}$ for $l_{1}, l_{2} \in L$. Moreover, since $\varepsilon \otimes i d: A \otimes L \rightarrow \mathbb{K} \otimes L$ is a $A$-Leibniz algebra homomorphism,

$$
(\varepsilon \otimes i d)\left[1 \otimes l_{1}, 1 \otimes l_{2}\right]_{\lambda}=\left[l_{1}, l_{2}\right]=(\varepsilon \otimes i d)\left(1 \otimes\left[l_{1}, l_{2}\right]\right)
$$


which implies

$$
\left[1 \otimes l_{1}, 1 \otimes l_{2}\right]_{\lambda}-1 \otimes\left[l_{1}, l_{2}\right] \in \operatorname{ker}(\varepsilon \otimes i d) .
$$

Hence we can write

$$
\left[1 \otimes l_{1}, 1 \otimes l_{2}\right]_{\lambda}=1 \otimes\left[l_{1}, l_{2}\right]+\sum_{j} c_{j} \otimes y_{j}
$$

where $\sum_{j} c_{j} \otimes y_{j}$ is a finite sum with $c_{j} \in \operatorname{ker}(\varepsilon)=\mathfrak{M}$ and $y_{j} \in L$.

Definition 3.2. Let $A$ be a complete local algebra $\left(A=\lim _{n \rightarrow \infty}\left(A / \mathfrak{M}^{n}\right)\right.$ ), where $\mathfrak{M}$ is the maximal ideal in $A$. A formal deformation of $\stackrel{n \rightarrow \infty}{L}$ with base $A$ is a A-Leibniz algebra structure on the completed tensor product

$$
A \hat{\otimes} L=\lim _{n \rightarrow \infty}\left(\left(A / \mathfrak{M}^{n}\right) \otimes L\right),
$$

which is the projective limit of deformations with base $A / \mathfrak{M}^{n}$ such that

$$
\varepsilon \otimes i d: A \hat{\otimes} L \rightarrow K \otimes L=L
$$

is a A-Leibniz algebra homomorphism.

Definition 3.3. Suppose $\lambda_{1}$ and $\lambda_{2}$ are two deformations of a Leibniz algebra $L$ with base $A$. We call them equivalent if there exists a Leibniz algebra isomorphism

$$
\phi:\left(A \otimes L,[,]_{\lambda_{1}}\right) \rightarrow\left(A \otimes L,[,]_{\lambda_{2}}\right)
$$

such that $(\varepsilon \otimes i d) \circ \phi=\varepsilon \otimes i d$.

We write $\lambda_{1} \cong \lambda_{2}$ if $\lambda_{1}$ is equivalent to $\lambda_{2}$.

Example 3.4. If $A=\mathbb{K}[[t]]$ then a formal deformation of a Leibniz algebra $L$ over $A$ is precisely a formal 1-parameter deformation of L(see [3]).

Definition 3.5. Suppose $\lambda$ is a given deformation of $L$ with base $(A, \mathfrak{M})$ and augmentation $\varepsilon: A \rightarrow \mathbb{K}$. Let $A^{\prime}$ be another commutative algebra with identity and a fixed augmentation $\varepsilon^{\prime}: A^{\prime} \rightarrow \mathbb{K}$. Suppose $\phi: A \rightarrow A^{\prime}$ is an algebra homomorphism with $\phi(1)=1$ and $\varepsilon^{\prime} \circ \phi=\varepsilon$. Let $\operatorname{ker}\left(\varepsilon^{\prime}\right)=\mathfrak{M}^{\prime}$. Then the push-out $\phi_{*} \lambda$ is the deformation of $L$ with base $\left(A^{\prime}, \mathfrak{M}^{\prime}\right)$ and bracket

$$
\left[a_{1}^{\prime} \otimes_{A}\left(a_{1} \otimes l_{1}\right), a_{2}^{\prime} \otimes_{A}\left(a_{2} \otimes l_{2}\right)\right]_{\phi_{*} \lambda}=a_{1}^{\prime} a_{2}^{\prime} \otimes_{A}\left[a_{1} \otimes l_{1}, a_{2} \otimes l_{2}\right]_{\lambda}
$$

where $a_{1}^{\prime}, a_{2}^{\prime} \in A^{\prime}, a_{1}, a_{2} \in A$ and $l_{1}, l_{2} \in L$. Here $A^{\prime}$ is considered as an $A$-module by the map $a^{\prime} \cdot a=a^{\prime} \phi(a)$ so that

$$
A^{\prime} \otimes L=\left(A^{\prime} \otimes_{A} A\right) \otimes L=A^{\prime} \otimes_{A}(A \otimes L) .
$$


Remark 3.6. If the bracket $[,]_{\lambda}$ is given by

$$
\left[1 \otimes l_{1}, 1 \otimes l_{2}\right]_{\lambda}=1 \otimes\left[l_{1}, l_{2}\right]+\sum_{j} c_{j} \otimes y_{j} \text { for } c_{j} \in \mathfrak{M} \text { and } y_{j} \in L
$$

then the bracket $[,]_{\phi_{*} \lambda}$ can be written as

$$
\left[1 \otimes l_{1}, 1 \otimes l_{2}\right]_{\phi_{*} \lambda}=1 \otimes\left[l_{1}, l_{2}\right]+\sum_{j} \phi\left(c_{j}\right) \otimes y_{j} .
$$

\section{Universal Infinitesimal Deformation}

In this section we construct a specific infinitesimal deformation of a Leibniz algebra $L$, which turns out to be universal in the class of all infinitesimal deformations of $L$. Let $L$ be a Leibniz algebra which satisfies the condition $\operatorname{dim}\left(H L^{2}(L ; L)\right)<\infty$. This is true for example, if $L$ is finite dimensional. Let us denote the space $H L^{2}(L ; L)$ by $\mathbb{H}$ throughout the paper. Consider the algebra $C_{1}=\mathbb{K} \oplus \mathbb{H}^{\prime}$ by setting $\left(k_{1}, h_{1}\right) \cdot\left(k_{2}, h_{2}\right)=\left(k_{1} k_{2}, k_{1} h_{2}+k_{2} h_{1}\right)$ where $\mathbb{H}^{\prime}$ is the dual of $\mathbb{H}$. Observe that the second summand is an ideal of $C_{1}$ with zero multiplication. Fix a homomorphism

$$
\mu: \mathbb{H} \longrightarrow C L^{2}(L ; L)=H o m\left(L^{\otimes 2} ; L\right)
$$

which takes a cohomology class into a cocycle representing it. Notice that there is an isomorphism $\mathbb{H}^{\prime} \otimes L \cong \operatorname{Hom}(\mathbb{H} ; L)$, so we have

$$
C_{1} \otimes L=\left(\mathbb{K} \oplus \mathbb{H}^{\prime}\right) \otimes L=(\mathbb{K} \otimes L) \oplus\left(\mathbb{H}^{\prime} \otimes L\right)=L \oplus \operatorname{Hom}(\mathbb{H} ; L) .
$$

Using the above identification, define a Leibniz bracket on $C_{1} \otimes L$ as follows. For $\left(l_{1}, \phi_{1}\right),\left(l_{2}, \phi_{2}\right) \in L \oplus \operatorname{Hom}(\mathbb{H} ; L)$ let

$$
\left[\left(l_{1}, \phi_{1}\right),\left(l_{2}, \phi_{2}\right)\right]=\left(\left[l_{1}, l_{2}\right], \psi\right)
$$

where the map $\psi: \mathbb{H} \longrightarrow L$ is given by

$$
\psi(\alpha)=\mu(\alpha)\left(l_{1}, l_{2}\right)+\left[\phi_{1}(\alpha), l_{2}\right]+\left[l_{1}, \phi_{2}(\alpha)\right] \text { for } \alpha \in \mathbb{H} .
$$

It is straightforward to check that $C_{1} \otimes L$ along with the above bracket is a Leibniz algebra over $C_{1}$. The Leibniz identity is a consequence of the fact that $\delta \mu(\alpha)=0$ for $\alpha \in \mathbb{H}$. Hence we get an infinitesimal deformation of $L$ with base $C_{1}=\mathbb{K} \oplus \mathbb{H}^{\prime}$.

Proposition 4.1. Up to an isomorphism, this deformation does not depend on the choice of $\mu$. 
Proof. Let

$$
\mu^{\prime}: \mathbb{H} \longrightarrow C L^{2}(L ; L)
$$

be another choice for $\mu$. Then for $\alpha \in \mathbb{H}$, the cochains $\mu(\alpha)$ and $\mu^{\prime}(\alpha)$ in $C L^{2}(L ; L)$ represent the same class $\alpha$. So $\mu(\alpha)-\mu^{\prime}(\alpha)$ is a coboundary. Hence we can define a homomorphism

$$
\gamma: \mathbb{H} \longrightarrow C L^{1}(L ; L)
$$

on a basis $\left\{h_{i}\right\}_{1 \leq i \leq n}$ of $\mathbb{H}$ by $\gamma\left(h_{i}\right)=\gamma_{i}$ with $\delta \gamma_{i}=\mu\left(h_{i}\right)-\mu^{\prime}\left(h_{i}\right)$. Clearly, $\mu^{\prime}-\mu=\delta \gamma$.

Using the identification $C_{1} \otimes L \cong L \oplus H o m(\mathbb{H} ; L)$, define

$$
\rho: C_{1} \otimes L \longrightarrow C_{1} \otimes L \text { by } \rho((l, \phi))=(l, \psi),
$$

where $\psi(\alpha)=\phi(\alpha)+\gamma(\alpha)(l), l \in L$ and $\phi \in \operatorname{Hom}(\mathbb{H} ; L)$.

It is routine to check that $\rho$ is a $C_{1}$-linear automorphism of $C_{1} \otimes L$, where $\rho^{-1}(l, \psi)=(l, \phi)$ with $\phi(\alpha)=\psi(\alpha)-\gamma(\alpha)(l)$ for $\alpha \in \mathbb{H}$.

It remains to show that $\rho$ preserves the bracket. Let $\left(l_{1}, \phi_{1}\right),\left(l_{2}, \phi_{2}\right) \in C_{1} \otimes L$ with $\rho\left(l_{1}, \phi_{1}\right)=\left(l_{1}, \psi_{1}\right)$ and $\rho\left(l_{2}, \phi_{2}\right)=\left(l_{2}, \psi_{2}\right)$. Suppose

$\left[\left(l_{1}, \phi_{1}\right),\left(l_{2}, \phi_{2}\right)\right]=\left(\left[l_{1}, l_{2}\right], \phi_{3}\right)$

where $\phi_{3}(\alpha)=\mu(\alpha)\left(l_{1}, l_{2}\right)+\left[\phi_{1}(\alpha), l_{2}\right]+\left[l_{1}, \phi_{2}(\alpha)\right]$, and

$\left[\left(l_{1}, \psi_{1}\right),\left(l_{2}, \psi_{2}\right)\right]=\left(\left[l_{1}, l_{2}\right], \psi_{3}\right)$

where $\psi_{3}(\alpha)=\mu^{\prime}(\alpha)\left(l_{1}, l_{2}\right)+\left[\psi_{1}(\alpha), l_{2}\right]+\left[l_{1}, \psi_{2}(\alpha)\right]$.

Then $\psi_{3}(\alpha)=\mu^{\prime}(\alpha)\left(l_{1}, l_{2}\right)+\left[\psi_{1}(\alpha), l_{2}\right]+\left[l_{1}, \psi_{2}(\alpha)\right]$

$$
\begin{aligned}
= & \mu(\alpha)\left(l_{1}, l_{2}\right)-\delta \gamma(\alpha)\left(l_{1}, l_{2}\right)+\left[\phi_{1}(\alpha)+\gamma(\alpha)\left(l_{1}\right), l_{2}\right] \\
& +\left[l_{1}, \phi_{2}(\alpha)+\gamma(\alpha)\left(l_{2}\right)\right] \\
= & \mu(\alpha)\left(l_{1}, l_{2}\right)-\left[l_{1}, \gamma(\alpha)\left(l_{2}\right)\right]-\left[\gamma(\alpha)\left(l_{1}\right), l_{2}\right]+\gamma(\alpha)\left(\left[l_{1}, l_{2}\right]\right) \\
+ & {\left[\phi_{1}(\alpha), l_{2}\right]+\left[\gamma(\alpha)\left(l_{1}\right), l_{2}\right]+\left[\phi_{1}(\alpha), l_{2}\right]+\left[l_{1}, \phi_{2}(\alpha)\right]+\left[l_{1}, \gamma(\alpha)\left(l_{2}\right)\right] } \\
= & \mu(\alpha)\left(l_{1}, l_{2}\right)+\left[\phi_{1}(\alpha), l_{2}\right]+\left[l_{1}, \phi_{2}(\alpha)\right]+\gamma(\alpha)\left(\left[l_{1}, l_{2}\right]\right) \\
= & \phi_{3}(\alpha)+\gamma(\alpha)\left(\left[l_{1}, l_{2}\right]\right) .
\end{aligned}
$$

Hence $\rho\left(\left[l_{1}, l_{2}\right], \phi_{3}\right)=\left(\left[l_{1}, l_{2}\right], \psi_{3}\right)=\left[\left(l_{1}, \psi_{1}\right),\left(l_{2}, \psi_{2}\right)\right]=\left[\rho\left(l_{1}, \phi_{1}\right), \rho\left(l_{2}, \phi_{2}\right)\right]$.

Therefore, up to an isomorphism, the infinitesimal deformation obtained above is independent of the choice of $\mu$.

We shall denote this deformation of $L$ by $\eta_{1}$. 
Remark 4.2. Suppose $\left\{h_{i}\right\}_{1 \leq i \leq n}$ is a basis of $\mathbb{H}$ and $\left\{g_{i}\right\}_{1 \leq i \leq n}$ is the dual basis. Let $\mu\left(h_{i}\right)=\mu_{i} \in C L^{2}(L ; L)$. Under the identification $C_{1} \otimes L=L \oplus H o m(\mathbb{H} ; L)$, an element $(l, \phi) \in L \oplus H o m(\mathbb{H} ; L)$ corresponds to $1 \otimes l+\sum_{i=1}^{n} g_{i} \otimes \phi\left(h_{i}\right)$. Then for $\left(l_{1}, \phi_{1}\right),\left(l_{2}, \phi_{2}\right) \in L \oplus H o m(H ; L)$ their bracket $\left(\left[l_{1}, l_{2}\right], \psi\right)$ corresponds to

$$
1 \otimes\left[l_{1}, l_{2}\right]+\sum_{i=1}^{n} g_{i} \otimes\left(\mu_{i}\left(l_{1}, l_{2}\right)+\left[\phi_{1}\left(h_{i}\right), l_{2}\right]+\left[l_{1}, \phi_{2}\left(h_{i}\right)\right]\right) .
$$

In particular, for $l_{1}, l_{2} \in L$ we have

$$
\left[1 \otimes l_{1}, 1 \otimes l_{2}\right]_{\eta_{1}}=1 \otimes\left[l_{1}, l_{2}\right]+\sum_{i=1}^{n} g_{i} \otimes \mu_{i}\left(l_{1}, l_{2}\right) .
$$

The main property of $\eta_{1}$ is the universality in the class of infinitesimal deformations with a finite dimensional base (Proposition 4.4).

Let $\lambda$ be an infinitesimal deformation of the Leibniz algebra $L$ with a finite dimensional base $A$. Let $\left\{m_{i}\right\}_{1 \leq i \leq r}$ be a basis of $\mathfrak{M}=\operatorname{ker}(\varepsilon)$ and $\left\{\xi_{i}\right\}_{1 \leq i \leq r}$ be the dual basis. Note that any element $\xi \in \mathfrak{M}^{\prime}$ can be viewed as an element in the dual space $A^{\prime}$ with $\xi(1)=0$. For any such $\xi$ set

$$
\alpha_{\lambda, \xi}\left(l_{1}, l_{2}\right)=\xi \otimes i d\left(\left[1 \otimes l_{1}, 1 \otimes l_{2}\right]_{\lambda}\right) \quad \text { for } l_{1}, l_{2} \in L .
$$

This defines a cochain $\alpha_{\lambda, \xi} \in \operatorname{Hom}\left(L^{\otimes 2} ; L\right)=C L^{2}(L ; L)$.

If we set $\psi_{i}=\alpha_{\lambda, \xi_{i}}$ for $1 \leq i \leq r$, the Leibniz bracket (11) in terms of the basis of $\mathfrak{M}$ takes the form

$$
\begin{aligned}
{\left[1 \otimes l_{1}, 1 \otimes l_{2}\right]_{\lambda} } & =1 \otimes\left[l_{1}, l_{2}\right]+\sum_{i=1}^{r} m_{i} \otimes x_{i} \\
& =1 \otimes\left[l_{1}, l_{2}\right]+\sum_{i=1}^{r} m_{i} \otimes \psi_{i}\left(l_{1}, l_{2}\right) .
\end{aligned}
$$

Lemma 4.3. The cochain $\alpha_{\lambda, \xi} \in C L^{2}(L ; L)$ is a cocycle.

Proof. By definition,

$$
\begin{aligned}
& \delta \alpha_{\lambda, \xi}\left(l_{1}, l_{2}, l_{3}\right) \\
= & {\left[l_{1}, \alpha_{\lambda, \xi}\left(l_{2}, l_{3}\right)\right]+\left[\alpha_{\lambda, \xi}\left(l_{1}, l_{3}\right), l_{2}\right]-\left[\alpha_{\lambda, \xi}\left(l_{1}, l_{2}\right), l_{3}\right] } \\
& -\alpha_{\lambda, \xi}\left(\left[l_{1}, l_{2}\right], l_{3}\right)+\alpha_{\lambda, \xi}\left(\left[l_{1}, l_{3}\right], l_{2}\right)+\alpha_{\lambda, \xi}\left(l_{1},\left[l_{2}, l_{3}\right]\right) \text { for } l_{1}, l_{2}, l_{3} \in L .
\end{aligned}
$$

Observe that

$$
\begin{aligned}
& (\xi \otimes i d)\left(\left[1 \otimes l_{1},\left[1 \otimes l_{2}, 1 \otimes l_{3}\right]_{\lambda}\right]_{\lambda}\right) \\
& =(\xi \otimes i d)\left(\left[1 \otimes l_{1}, 1 \otimes\left[l_{2}, l_{3}\right]\right]_{\lambda}+\left[1 \otimes l_{1}, \sum_{j=1}^{r} m_{j} \otimes x_{j}\right]_{\lambda}\right)(\text { using (4) }) \\
& =\alpha_{\lambda, \xi}\left(l_{1},\left[l_{2}, l_{3}\right]\right)+\sum_{j=1}^{r}(\xi \otimes i d)\left[1 \otimes l_{1}, m_{j} \otimes x_{j}\right]_{\lambda} .
\end{aligned}
$$


Moreover,

$$
\begin{aligned}
(\xi \otimes i d)\left[1 \otimes l_{1}, m_{j} \otimes x_{j}\right]_{\lambda} & =(\xi \otimes i d) m_{j}\left[1 \otimes l_{1}, 1 \otimes x_{j}\right]_{\lambda} \\
& =(\xi \otimes i d) m_{j}\left(1 \otimes\left[l_{1}, x_{j}\right]+\sum_{i=1}^{r} m_{i} \otimes x_{j i}\right) \\
& =(\xi \otimes i d)\left(m_{j} \otimes\left[l_{1}, x_{j}\right]\right) \quad\left(\mathfrak{M}^{2}=0\right) \\
& =\left[l_{1},(\xi \otimes i d)\left(m_{j} \otimes x_{j}\right)\right] .
\end{aligned}
$$

Therefore

$$
\begin{aligned}
& (\xi \otimes i d)\left(\left[1 \otimes l_{1},\left[1 \otimes l_{2}, 1 \otimes l_{3}\right]_{\lambda}\right]_{\lambda}\right) \\
& =\alpha_{\lambda, \xi}\left(l_{1},\left[l_{2}, l_{3}\right]\right)+\left[l_{1},(\xi \otimes i d) \sum_{j=1}^{r} m_{j} \otimes x_{j}\right] \\
& =\alpha_{\lambda, \xi}\left(l_{1},\left[l_{2}, l_{3}\right]\right)+\left[l_{1},(\xi \otimes i d)\left(\left[1 \otimes l_{2}, 1 \otimes l_{3}\right]_{\lambda}-1 \otimes\left[l_{2}, l_{3}\right]\right)\right] \quad(\text { by using (44) }) \\
& =\alpha_{\lambda, \xi}\left(l_{1},\left[l_{2}, l_{3}\right]\right)+\left[l_{1}, \alpha_{\lambda, \xi}\left(l_{2}, l_{3}\right)\right] \quad(\xi(1)=0) .
\end{aligned}
$$

Similarly,

$$
\begin{aligned}
& (\xi \otimes i d)\left(\left[\left[1 \otimes l_{1}, 1 \otimes l_{2}\right]_{\lambda}, 1 \otimes l_{3}\right]_{\lambda}\right)=\alpha_{\lambda, \xi}\left(\left[l_{1}, l_{2}\right], l_{3}\right)+\left[\alpha_{\lambda, \xi}\left(l_{1}, l_{2}\right), l_{3}\right], \\
& (\xi \otimes i d)\left(\left[\left[1 \otimes l_{1}, 1 \otimes l_{3}\right]_{\lambda}, 1 \otimes l_{2}\right]_{\lambda}\right)=\alpha_{\lambda, \xi}\left(\left[l_{1}, l_{3}\right], l_{2}\right)+\left[\alpha_{\lambda, \xi}\left(l_{1}, l_{3}\right), l_{2}\right] .
\end{aligned}
$$

It follows that

$$
\begin{aligned}
\delta \alpha_{\lambda, \xi}\left(l_{1}, l_{2}, l_{3}\right)= & (\xi \otimes i d)\left(\left[1 \otimes l_{1},\left[1 \otimes l_{2}, 1 \otimes l_{3}\right]_{\lambda}\right]_{\lambda}-\left[\left[1 \otimes l_{1}, 1 \otimes l_{2}\right]_{\lambda}, 1 \otimes l_{3}\right]_{\lambda}\right. \\
& \left.+\left[\left[1 \otimes l_{1}, 1 \otimes l_{3}\right]_{\lambda}, 1 \otimes l_{2}\right]_{\lambda}\right) \\
= & 0 \quad \text { (by the Leibniz relation). }
\end{aligned}
$$

Proposition 4.4. For any infinitesimal deformation $\lambda$ of a Leibniz algebra $L$ with a finite dimensional base $A$ there exists a unique homomorphism $\phi: C_{1}=$ $\left(\mathbb{K} \oplus \mathbb{H}^{\prime}\right) \longrightarrow A$ such that $\lambda$ is equivalent to the push-out $\phi_{*} \eta_{1}$.

Proof. Let $\lambda$ be an infinitesimal deformation of a Leibniz algebra $L$ with base $A$, where $A$ is a finite dimensional local algebra over $\mathbb{K}$ and $\mathfrak{M}$ is the maximal ideal in $A$. Let $\operatorname{dim}(\mathfrak{M})=r$. Suppose $\left\{m_{i}\right\}_{1 \leq i \leq r}$ is a basis of $\mathfrak{M}$ and $\left\{\xi_{i}\right\}_{1 \leq i \leq r}$ be the corresponding dual basis of $\mathfrak{M}^{\prime}$. For $\xi_{i} \in \mathfrak{M}^{\prime}$ let $a_{\lambda, \xi_{i}} \in \mathbb{H}$ be the cohomology class of the cocycle $\alpha_{\lambda, \xi_{i}}$. The correspondences

$$
\xi_{i} \longmapsto \alpha_{\lambda, \xi_{i}} \text { and } \xi_{i} \longmapsto a_{\lambda, \xi_{i}}
$$


for $1 \leq i \leq r$ define homomorphisms

$$
\alpha_{\lambda}: \mathfrak{M}^{\prime} \longrightarrow C L^{2}(L ; L) \text { with } \delta \circ \alpha_{\lambda}=0 \text { and } a_{\lambda}: \mathfrak{M}^{\prime} \longrightarrow \mathbb{H}
$$

We claim that

(a) Two deformations $\lambda_{1}$ and $\lambda_{2}$ are equivalent if and only if $a_{\lambda_{1}}=a_{\lambda_{2}}$.

(b) If $\phi=i d \oplus a_{\lambda}^{\prime}: C_{1} \longrightarrow \mathbb{K} \oplus \mathfrak{M}=A$ then $\phi_{*} \eta_{1}$ is equivalent to $\lambda$.

Let $\lambda_{1}$ and $\lambda_{2}$ be two equivalent deformations of the Leibniz algebra $L$ with base $A$. Then there exists a $A$-Leibniz algebra isomorphism

$$
\rho:\left(A \otimes L,[,]_{\lambda_{1}}\right) \longrightarrow\left(A \otimes L,[,]_{\lambda_{2}}\right) \text { with }(\varepsilon \otimes i d) \circ \rho=\varepsilon \otimes i d
$$

Now $A \otimes L=(\mathbb{K} \oplus \mathfrak{M}) \otimes L=(\mathbb{K} \otimes L) \oplus(\mathfrak{M} \otimes L)=L \oplus(\mathfrak{M} \otimes L)$. Thus any element of $A \otimes L$ is of the form $\left(l, \sum_{i=1}^{r} m_{i} \otimes l_{i}\right)$ where $l_{i} \in L$ for $1 \leq i \leq r$. By $A$-linearity, $\rho$ is determined by the values $\rho(1 \otimes l)$ for $l \in L$ and hence $\rho$ is of the form $\rho=\rho_{1}+\rho_{2}$ where $\rho_{1}: L \longrightarrow L$ and $\rho_{2}: L \longrightarrow \mathfrak{M} \otimes L$. The map $\rho_{1}$ must be the identity map $i d: L \longrightarrow L$ by the compatibility $(\varepsilon \otimes i d) \circ \rho=\varepsilon \otimes i d$. We shall use the following standard identifications.

$$
\operatorname{Hom}(L ; \mathfrak{M} \otimes L) \cong \mathfrak{M} \otimes \operatorname{Hom}(L ; L) \cong H o m\left(\mathfrak{M}^{\prime} ; H o m(L ; L)\right)
$$

In terms of bases of $\mathfrak{M}$ and $\mathfrak{M}^{\prime}$, the above isomorphisms are given as follows.

$$
\rho_{2} \longmapsto \sum_{i=1}^{r} m_{i} \otimes \phi_{i} \longmapsto \sum_{i=1}^{r} \chi_{i}
$$

where $\phi_{i}=\left(\xi_{i} \otimes i d\right) \circ \rho_{2}$ and $\chi_{i}\left(\xi_{j}\right)=\delta_{i, j} \phi_{i}$. We have

$$
\rho(1 \otimes l)=\rho_{1}(1 \otimes l)+\rho_{2}(1 \otimes l)=1 \otimes l+\sum_{i=1}^{r} m_{i} \otimes \phi_{i}(l) \text { for } l \in L .
$$

The map $\rho$ is a Leibniz algebra homomorphism if and only if

$$
\rho\left(\left[1 \otimes l_{1}, 1 \otimes l_{2}\right]_{\lambda_{1}}\right)=\left[\rho\left(1 \otimes l_{1}\right), \rho\left(1 \otimes l_{2}\right)\right]_{\lambda_{2}}
$$

If we take $\psi_{i}{ }^{k}=\alpha_{\lambda_{k}, \xi_{i}} 1 \leq i \leq r$ for $k=1$ and 2 , we have

$$
\left[1 \otimes l_{1}, 1 \otimes l_{2}\right]_{\lambda_{k}}=1 \otimes\left[l_{1}, l_{2}\right]+\sum_{i=1}^{r} m_{i} \otimes \psi_{i}{ }^{k}\left(l_{1}, l_{2}\right)
$$


Therefore $\rho\left(\left[1 \otimes l_{1}, 1 \otimes l_{2}\right]_{\lambda_{1}}\right)$

$$
\begin{aligned}
& =1 \otimes\left[l_{1}, l_{2}\right]+\sum_{i=1}^{r} m_{i} \otimes \phi_{i}\left(\left[l_{1}, l_{2}\right]\right) \\
& +\sum_{i=1}^{r} m_{i}\left(1 \otimes \psi_{i}^{1}\left(l_{1}, l_{2}\right)+\sum_{j=1}^{r} m_{j} \otimes \phi_{j}\left(\psi_{i}{ }^{1}\left(l_{1}, l_{2}\right)\right)\right) \\
& =1 \otimes\left[l_{1}, l_{2}\right]+\sum_{i=1}^{r} m_{i} \otimes \phi_{i}\left(\left[l_{1}, l_{2}\right]\right)+\sum_{i=1}^{r} m_{i}\left(1 \otimes \psi_{i}{ }^{1}\left(l_{1}, l_{2}\right)\right) \\
& \quad\left(m_{i} m_{j}=0\right) .
\end{aligned}
$$

Similarly, $\left[\rho\left(1 \otimes l_{1}\right), \rho\left(1 \otimes l_{2}\right)\right]_{\lambda_{2}}$

$$
\begin{aligned}
= & 1 \otimes\left[l_{1}, l_{2}\right]+\sum_{i=1}^{r} m_{i} \otimes \psi_{i}^{2}\left(l_{1}, l_{2}\right)+\sum_{i=1}^{r} m_{i} \otimes\left[l_{1}, \phi_{i}\left(l_{2}\right)\right] \\
& +\sum_{i=1}^{r} m_{i} \otimes\left[\phi_{i}\left(l_{1}\right), l_{2}\right] .
\end{aligned}
$$

Thus, $\left[\rho\left(1 \otimes l_{1}\right), \rho\left(1 \otimes l_{2}\right)\right]_{\lambda_{2}}-\rho\left(\left[1 \otimes l_{1}, 1 \otimes l_{2}\right]_{\lambda_{1}}\right)=0$

$$
\begin{aligned}
& \Leftrightarrow \sum_{i=1}^{r} m_{i} \otimes\left(\psi_{i}^{2}\left(l_{1}, l_{2}\right)-\psi_{i}^{1}\left(l_{1}, l_{2}\right)\right)+\sum_{i=1}^{r} m_{i} \otimes \delta \phi_{i}\left(l_{1}, l_{2}\right)=0 \\
& \Leftrightarrow \psi_{i}^{1}\left(l_{1}, l_{2}\right)-\psi_{i}^{2}\left(l_{1}, l_{2}\right)=\delta \phi_{i}\left(l_{1}, l_{2}\right) \\
& \text { that is, } \alpha_{\lambda_{1}, \xi_{i}}-\alpha_{\lambda_{2}, \xi_{i}}=\delta \phi_{i} \text { for } 1 \leq i \leq r \\
& \Leftrightarrow a_{\lambda_{1}}=a_{\lambda_{2}} .
\end{aligned}
$$

This proves (a).

Now consider the map

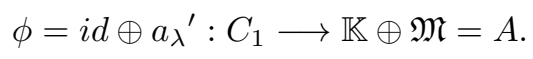

By (a) it is enough to show that $\alpha_{\phi_{*} \eta_{1}}=\mu \circ a_{\lambda}$. Let $\left\{h_{i}\right\}_{1 \leq i \leq n}$ be a basis of $\mathbb{H}$ and $\left\{g_{i}\right\}_{1 \leq i \leq n}$ be the corresponding dual basis of $\mathbb{H}^{\prime}$. By Remarks 3.6 and 4.2 we have

$$
\left[1 \otimes l_{1}, 1 \otimes l_{2}\right]_{\phi_{*} \eta_{1}}=1 \otimes\left[l_{1}, l_{2}\right]+\sum_{i=1}^{n} \phi\left(g_{i}\right) \otimes \mu\left(h_{i}\right)\left(l_{1}, l_{2}\right) .
$$

Let $a_{\lambda}{ }^{\prime}: \mathbb{H}^{\prime} \longrightarrow \mathfrak{M}$ be the dual of $a_{\lambda}$. Then

$$
a_{\lambda}{ }^{\prime}\left(g_{j}\right)=\sum_{i=1}^{r} \xi_{i}\left(a_{\lambda}{ }^{\prime}\left(g_{j}\right)\right) m_{i} \text { and } a_{\lambda}\left(\xi_{i}\right)=\sum_{j=1}^{n} g_{j}\left(a_{\lambda}\left(\xi_{i}\right)\right) h_{j} .
$$




$$
\text { Thus } \begin{aligned}
\alpha_{\phi_{*} \eta_{1}}\left(\xi_{i}\right)\left(l_{1}, l_{2}\right) & =\xi_{i} \otimes i d\left[1 \otimes l_{1}, 1 \otimes l_{2}\right]_{\phi_{*} \eta_{1}} \\
& =\xi_{i} \otimes i d\left(1 \otimes\left[l_{1}, l_{2}\right]+\sum_{j=1}^{n} \phi\left(g_{j}\right) \otimes \mu\left(h_{j}\right)\left(l_{1}, l_{2}\right)\right) \\
& =\xi_{i} \otimes i d\left(\sum_{j=1}^{n} a_{\lambda}{ }^{\prime}\left(g_{j}\right) \otimes \mu\left(h_{j}\right)\left(l_{1}, l_{2}\right)\right) \\
& \left.=\sum_{j=1}^{n} \xi_{i}\left(a_{\lambda}{ }^{\prime}\left(g_{j}\right)\right) \otimes \mu\left(h_{j}\right)\left(l_{1}, l_{2}\right)\right) \\
& \left.=\sum_{j=1}^{n} g_{j}\left(a_{\lambda}\left(\xi_{i}\right)\right) \otimes\left(h_{i}\right)\left(l_{1}, l_{2}\right)\right) \\
& =\mu\left(\sum_{j=1}^{n} g_{j}\left(a_{\lambda}\left(\xi_{i}\right)\right) h_{j}\right)\left(l_{1}, l_{2}\right) \\
& =\mu \circ a_{\lambda}\left(\xi_{i}\right)\left(l_{1}, l_{2}\right) .
\end{aligned}
$$

The uniqueness part of the theorem follows from the definition of $\phi$.

Suppose $A$ is a local algebra with the unique maximal ideal $\mathfrak{M}$ and $\pi: A \rightarrow$ $A / \mathfrak{M}^{2}$ the corresponding quotient map. Assume $\operatorname{dim}\left(A / \mathfrak{M}^{2}\right)$ is finite. The algebra $A / \mathfrak{M}^{2}$ is obviously local with maximal ideal $\mathfrak{M} / \mathfrak{M}^{2}$ and $\left(\mathfrak{M} / \mathfrak{M}^{2}\right)^{2}=0$. If $\lambda$ is a deformation of $L$ with base $A$ then $\pi_{*} \lambda$ is a deformation with base $A / \mathfrak{M}^{2}$ and it is clearly infinitesimal. Therefore by the previous proposition we have a map

$$
a_{\pi * \lambda}:\left(\mathfrak{M} / \mathfrak{M}^{2}\right)^{\prime} \rightarrow \mathbb{H} .
$$

Definition 4.5. The dual space $\left(\mathfrak{M} / \mathfrak{M}^{2}\right)^{\prime}$ is called the tangent space of $A$ and is denoted by TA. The map $a_{\pi * \lambda}$ is called the differential of $\lambda$ and is denoted by $d \lambda$.

It follows from Proposition 4.4 that equivalent deformations have the same differential. We have constructed in this section the universal infinitesimal deformation and our goal is to extend it to a versal one. It is known that in the category of deformations of an algebraic object generally there is no universal deformation [13. But under certain natural conditions it is possible to get a "versal" object, which still induces all non-equivalent deformations.

Definition 4.6. A formal deformation $\eta$ of a Leibniz algebra $L$ with base $C$ is called versal, if

(i) for any formal deformation $\lambda$ of $L$ with base $A$ there exists a homomorphism $f: C \rightarrow A$ such that the deformation $\lambda$ is equivalent to $f_{*} \eta$;

(ii) if A satisfies the condition $\mathfrak{M}^{2}=0$, then $f$ is unique. 
Theorem 4.7. If $\mathbb{H}$ is finite dimensional, then there exists a versal deformation.

Proof. Follows from the general theorem of Schlessinger [22, like it was shown for Lie algebras in [4.

\section{Obstructions}

The aim of this section is to study obstructions in extending deformations. For this we need the interpretation of 1- and 2-dimensional Harrison cohomology of a commutative algebra. Let us recall some definitions and results from [12.

Let $A$ be a commutative algebra with 1 over $\mathbb{K}$. Let $\left(C_{q}(A), \delta\right)$ denote the standard Hochschild complex, where $C_{q}(A)$ is the $A$-module $A^{\otimes(q+1)}$ with $A$ acting on the first factor by multiplication of $A$. Let $S h_{q}(A)$ be the $A$-submodule of $C_{q}(A)$ generated by chains

$$
=\sum_{\left(i_{1}, i_{2}, \ldots, i_{q}\right) \in S h(p, q-p)} \operatorname{sgn}\left(i_{1}, i_{2}, \ldots, i_{q}\right)\left(a_{i_{1}}, a_{i_{2}}, \ldots, a_{i_{q}}\right) \in C_{q}(A)
$$

for $a_{1}, a_{2}, \ldots, a_{q} \in A ; 0<p<q$.

It turns out that $S h_{*}$ is a subcomplex of $C_{*}(A)$ and hence we have a complex called the Harrison complex

$$
C h(A)=\left\{C h_{q}(A), \delta\right\} ; C h_{q}(A)=C_{q}(A) / S h_{q}(A) .
$$

For an $A$-module $M$, the Harrison cochain complex defining the Harrison cohomology with coefficients in $M$ is given by $C h^{q}(A ; M)=H o m\left(C h_{q}(A), M\right)$.

Definition 5.1. For an A-module $M$ we define

$$
H_{\text {Harr }}^{q}(A ; M)=H^{q}(\operatorname{Hom}(C h(A), M)) .
$$

Proposition 5.2. Let $A$ be a commutative local algebra with the maximal ideal $\mathfrak{M}$, and let $M$ be an A-module with $\mathfrak{M} M=0$. Then we have the canonical isomorphism

$$
H_{\text {Harr }}^{q}(A ; M) \cong H_{\text {Harr }}^{q}(A ; \mathbb{K}) \otimes M .
$$

Definition 5.3. An extension $B$ of an algebra $A$ by an $A$-module $M$ is a $\mathbb{K}$ algebra $B$ together with an exact sequence of $\mathbb{K}$-modules

$$
0 \longrightarrow M \stackrel{i}{\longrightarrow} B \stackrel{p}{\longrightarrow} A \longrightarrow 0,
$$

where $p$ is an $\mathbb{K}$-algebra homomorphism, and the $B$-module structure on $i(M)$ is given by the A-module structure of $M$ by $i(m) \cdot b=i(m p(b))$. 
Proposition 5.4. (i) The space $H_{H a r r}^{1}(A ; M)$ is isomorphic to the space of derivations $A \longrightarrow M$.

(ii) Elements of $H_{\text {Harr }}^{2}(A ; M)$ correspond bijectively to isomorphism classes of extensions

$$
0 \longrightarrow M \longrightarrow B \longrightarrow A \longrightarrow 0
$$

of the algebra $A$ by means of $M$.

(iii) The space $H_{H a r r}^{1}(A ; M)$ can also be interpreted as the group of automorphisms of any given extension of $A$ by $M$.

Corollory 5.5. If $A$ is a local algebra with the maximal ideal $\mathfrak{M}$, then

$$
H_{\text {Harr }}^{1}(A ; \mathbb{K}) \cong\left(\frac{\mathfrak{M}}{\mathfrak{M}^{2}}\right)^{\prime}=T A .
$$

Let $\lambda$ be a deformation of a Leibniz algebra $L$ with a finite dimensional local base $A$ and augmentation $\varepsilon$. Consider $[f] \in H_{\text {Harr }}^{2}(A ; \mathbb{K})$. Suppose

$$
0 \longrightarrow \mathbb{K} \stackrel{i}{\longrightarrow} B \stackrel{p}{\longrightarrow} A \longrightarrow 0
$$

is a representative of the class of 1 - dimensional extensions of $A$, corresponding to the cohomology class of $f$. Let $I=(i \otimes i d): L \cong \mathbb{K} \otimes L \longrightarrow B \otimes L$, $P=(p \otimes i d): B \otimes L \longrightarrow A \otimes L$ and $E=(\hat{\varepsilon} \otimes i d): B \otimes L \longrightarrow \mathbb{K} \otimes L \cong L$, where $\hat{\varepsilon}=\varepsilon \circ p$ is the augmentation of $B$ corresponding to the augmentation $\varepsilon$ of $A$. Fix a section $q: A \longrightarrow B$ of $p$ in the above extension, then

$$
b \longmapsto\left(p(b), i^{-1}(b-q \circ p(b))\right)
$$

is a $\mathbb{K}$ - module isomorphism $B \longrightarrow(A \oplus \mathbb{K})$. Let us denote by $(a, k)_{q} \in B$ the inverse of $(a, k) \in(A \oplus \mathbb{K})$ under the above isomorphism. The algebra structure of $B$ is determined by $f$ and is given by

$$
\left(a_{1}, k_{1}\right)_{q} \circ\left(a_{2}, k_{2}\right)_{q}=\left(a_{1} a_{2}, a_{1} \cdot k_{2}+a_{2} \cdot k_{1}+f\left(a_{1}, a_{2}\right)\right)_{q} .
$$

Suppose $\operatorname{dim}(A)=r+1$ and $\left\{m_{i}\right\}_{1 \leq i \leq r}$ is a basis of the maximal ideal $\mathfrak{M}_{A}$ of A. Then $\left\{n_{i}\right\}_{1 \leq i \leq r+1}$ is a basis of the maximal ideal $\mathfrak{M}_{B}=p^{-1}\left(\mathfrak{M}_{A}\right)$ of $B$, where $n_{j}=\left(m_{j}, 0\right)_{q}$, for $1 \leq j \leq r$ and $n_{r+1}=(0,1)_{q}$. Take the dual basis $\left\{\xi_{i}\right\}_{1 \leq i \leq r}$ of $\mathfrak{M}_{A}^{\prime}$. Then by (3i) and (4), we have 2-cochains $\psi_{i}=\alpha_{\lambda, \xi_{i}} \in C L^{2}(L ; L)$ for $1 \leq i \leq r$ such that $[,]_{\lambda}$ can be written as

$$
\left[1 \otimes l_{1}, 1 \otimes l_{2}\right]_{\lambda}=1 \otimes\left[l_{1}, l_{2}\right]+\sum_{i=1}^{r} m_{i} \otimes \psi_{i}\left(l_{1}, l_{2}\right) \text { for } l_{1}, l_{2} \in L .
$$


Let $\psi \in C L^{2}(L ; L)=H o m\left(L^{\otimes 2} ; L\right)$ be an arbitrary element. Define a $B$-bilinear operation $(B \otimes L)^{\otimes 2} \longrightarrow B \otimes L$,

$$
\left\{b_{1} \otimes l_{1}, b_{2} \otimes l_{2}\right\}=b_{1} b_{2} \otimes\left[l_{1}, l_{2}\right]+\sum_{j=1}^{r} b_{1} b_{2} n_{j} \otimes \psi_{j}\left(l_{1}, l_{2}\right)+b_{1} b_{2} n_{r+1} \psi\left(l_{1}, l_{2}\right) .
$$

It is routine to check that the $B$-bilinear map $\{$,$\} satisfies$

$$
\begin{aligned}
& \text { (i) } P\left\{l_{1}, l_{2}\right\}=\left[P\left(l_{1}\right), P\left(l_{2}\right)\right]_{\lambda} \text { for } l_{1}, l_{2} \in B \otimes L \\
& \text { (ii) }\left\{I(l), l_{1}\right\}=I\left[l, E\left(l_{1}\right)\right] \text { for } l \in L \text { and } l_{1} \in B \otimes L .
\end{aligned}
$$

So the Leibniz algebra structure $\lambda$ on $A \otimes L$ can be lifted to a $B$-bilinear operation $\{\}:,(B \otimes L)^{\otimes 2} \longrightarrow B \otimes L$ satisfying (7).

Define $\phi:(B \otimes L)^{\otimes 3} \longrightarrow B \otimes L$ by

$$
\phi\left(l_{1}, l_{2}, l_{3}\right)=\left\{l_{1},\left\{l_{2}, l_{3}\right\}\right\}-\left\{\left\{l_{1}, l_{2}\right\}, l_{3}\right\}+\left\{\left\{l_{1}, l_{3}\right\}, l_{2}\right\} \text { for } l_{1}, l_{2}, l_{3} \in B \otimes L .
$$

It is clear that $\{$,$\} satisfies the Leibniz relation if and only if \phi=0$. Now from property $(i)$ in (7) and the definition of $\phi$ it follows that $P \circ \phi\left(l_{1}, l_{2}, l_{3}\right)=$ 0 for $l_{1}, l_{2}, l_{3} \in B \otimes L$. Therefore $\phi$ takes values in $\operatorname{ker}(P)$. Observe that $\phi\left(l_{1}, l_{2}, l_{3}\right)=0$, whenever one of the arguments belongs to $\operatorname{ker}(E)$. Suppose $l_{1}=b \otimes l \in \operatorname{ker}(E) \subseteq B \otimes L$. Since $\operatorname{ker}(E)=\operatorname{ker}(\hat{\varepsilon}) \otimes L=p^{-1}(\operatorname{ker}(\varepsilon)) \otimes L=$ $\mathfrak{M}_{B} \otimes L$, we can write $l_{1}=\sum_{j=1}^{r+1} n_{j} \otimes l_{j}{ }^{\prime}$ with $l_{j}{ }^{\prime} \in L ; j=1, \ldots, r+1$. Then for $l_{2}, l_{3} \in B \otimes L$, we get

$$
\phi\left(l_{1}, l_{2}, l_{3}\right)=\phi\left(\sum_{j=1}^{r+1} n_{j} \otimes l_{j}^{\prime}, l_{2}, l_{3}\right)=\sum_{j=1}^{r+1} n_{j} \phi\left(l_{j}^{\prime}, l_{2}, l_{3}\right)=0 .
$$

This is because $\phi\left(l_{j}^{\prime}, l_{2}, l_{3}\right) \in \operatorname{ker}(P)=i m(I)=i m(i) \otimes L=i(\mathbb{K}) \otimes L$ and for any element $k \in \mathbb{K}$ and $l \in L$,

$$
\begin{aligned}
& n_{j} \cdot i(k) \otimes l=i\left(p\left(n_{j}\right) k\right) \otimes l=i\left(m_{j} \cdot k\right) \otimes l=i\left(\varepsilon\left(m_{j}\right) k\right) \otimes l=0 \quad \text { for } 1 \leq j \leq r \\
& \text { and } n_{r+1} \cdot i(k) \otimes l=k n_{r+1}^{2} \otimes l=0 \quad\left(m_{j} \in \mathfrak{M} \subset A \text { and } m_{j} \cdot k=\varepsilon\left(m_{j}\right) k\right) .
\end{aligned}
$$

The other two cases are similar. Thus $\phi$ defines a linear map

$$
\tilde{\phi}:\left(\frac{B \otimes L}{k e r(E)}\right)^{\otimes 3} \longrightarrow \operatorname{ker}(P),
$$

$\tilde{\phi}\left(b_{1} \otimes l_{1}+\operatorname{ker}(E), b_{2} \otimes l_{2}+\operatorname{ker}(E), b_{3} \otimes l_{3}+\operatorname{ker}(E)\right)=\phi\left(b_{1} \otimes l_{1}, b_{2} \otimes l_{2}, b_{3} \otimes l_{3}\right)$. Moreover, the surjective map $E: B \otimes L \longrightarrow \mathbb{K} \otimes L \cong L$, defined by $b \otimes l \longmapsto \hat{\varepsilon}(b) \otimes l$, induces an isomorphism $\frac{B \otimes L}{\operatorname{ker}(E)} \stackrel{\alpha}{\cong} L$, where

$$
\alpha: L \longrightarrow \frac{B \otimes L}{k e r(E)} ; \alpha(l)=1 \otimes l+\operatorname{ker}(E) .
$$


Also, $\operatorname{ker}(P)=i m(I)=i(\mathbb{K}) \otimes L=\mathbb{K} i(1) \otimes L \stackrel{\beta}{\cong} L$ where the isomorphism $\beta$ is given by $\beta\left(k n_{r+1} \otimes l\right)=k l$ with inverse $\beta^{-1}(l)=n_{r+1} \otimes l$. Thus we get a linear map $\bar{\phi}: L^{\otimes 3} \longrightarrow L$, such that $\bar{\phi}=\beta \circ \tilde{\phi} \circ \alpha^{\otimes 3}$. The cochains $\bar{\phi} \in C L^{3}(L ; L)$ and $\phi$ are related by

$$
n_{r+1} \otimes \bar{\phi}\left(l_{1}, l_{2}, l_{3}\right)=\phi\left(1 \otimes l_{1}, 1 \otimes l_{2}, 1 \otimes l_{3}\right) .
$$

We claim that the cochain $\bar{\phi}$ is a cocycle. The coboundary $\delta \bar{\phi}$ consists of 10 terms. Let us rewrite the first term of $\beta^{-1} \circ \delta \bar{\phi}$ as follows.

$$
\begin{aligned}
& \beta^{-1}\left(\left[l_{1}, \bar{\phi}\left(l_{2}, l_{3}, l_{4}\right)\right]\right) \\
= & n_{r+1} \otimes\left[l_{1}, \bar{\phi}\left(l_{2}, l_{3}, l_{4}\right)\right] \\
= & I\left(\left[l_{1}, \bar{\phi}\left(l_{2}, l_{3}, l_{4}\right)\right]\right) \quad\left(i(1)=n_{r+1}\right) \\
= & I\left(\left[l_{1}, E\left(1 \otimes \bar{\phi}\left(l_{2}, l_{3}, l_{4}\right)\right)\right]\right) \\
= & \left\{I\left(l_{1}\right), 1 \otimes \bar{\phi}\left(l_{2}, l_{3}, l_{4}\right)\right\} \quad(\text { by }(i i) \text { of (17) }) \\
= & \left\{n_{r+1} \otimes l_{1}, 1 \otimes \bar{\phi}\left(l_{2}, l_{3}, l_{4}\right)\right\} \\
= & \left\{1 \otimes l_{1}, n_{r+1} \otimes \bar{\phi}\left(l_{2}, l_{3}, l_{4}\right)\right\} \\
= & \left\{1 \otimes l_{1}, \phi\left(1 \otimes l_{2}, 1 \otimes l_{3}, 1 \otimes l_{4}\right)\right\} \quad(\text { by (9) }) \\
= & \left\{1 \otimes l_{1},\left\{1 \otimes l_{2},\left\{1 \otimes l_{3}, 1 \otimes l_{4}\right\}\right\}\right\}-\left\{1 \otimes l_{1},\left\{\left\{1 \otimes l_{2}, 1 \otimes l_{3}\right\}, 1 \otimes l_{4}\right\}\right\} \\
& +\left\{1 \otimes l_{1},\left\{\left\{1 \otimes l_{2}, 1 \otimes l_{4}\right\}, 1 \otimes l_{3}\right\}\right\} .
\end{aligned}
$$

Similarly, computing other terms and substituting in the expression of $\beta^{-1} \circ \delta \bar{\phi}$, we get $\beta^{-1} \circ \delta \bar{\phi}\left(l_{1}, l_{2}, l_{3}, l_{4}\right)=0$.

Let us show now that the cohomology class of $\bar{\phi}$ is independent of the choice of the lifting $\{$,$\} . Suppose \{$,$\} and \{,\}^{\prime}$ are two $B$-bilinear operations on $B \otimes$ $L$, lifting the Leibniz algebra structure $\lambda$ on $A \otimes L$. Let $\bar{\phi}$ and $\bar{\phi}^{\prime}$ be the corresponding cocycles. Set $\rho=\{,\}^{\prime}-\{$,$\} . Then \rho:(B \otimes L)^{\otimes 2} \longrightarrow B \otimes$ $L$ is a $B$-linear map. Observe that

$$
P \circ \rho\left(l_{1}, l_{2}\right)=\left[P\left(l_{1}\right), P\left(l_{2}\right)\right]_{\lambda}-\left[P\left(l_{1}\right), P\left(l_{2}\right)\right]_{\lambda}=0 \text { (by }(i) \text { in (17)). }
$$

Thus $\rho$ takes values in $\operatorname{ker}(P)$ and induces a linear map

$$
\begin{gathered}
\tilde{\rho}:\left(\frac{B \otimes L}{\operatorname{ker}(E)}\right)^{\otimes 2} \longrightarrow \operatorname{ker}(P), \\
\tilde{\rho}\left(l_{1}+\operatorname{ker}(E), l_{2}+\operatorname{ker}(E)\right)=\rho\left(l_{1}, l_{2}\right) \text { for } l_{1}, l_{2} \in B \otimes L .
\end{gathered}
$$

Hence we get a 2-cochain $\bar{\rho}: L^{\otimes 2} \longrightarrow L$ such that $\bar{\rho}=\beta \circ \tilde{\rho} \circ \alpha^{\otimes 2} \in C L^{2}(L ; L)$. As before, for $l_{1}, l_{2} \in L$, we have $n_{r+1} \otimes \bar{\rho}\left(l_{1}, l_{2}\right)=\rho\left(1 \otimes l_{1}, 1 \otimes l_{2}\right)$. Then a 
straightforward computation yields

$$
\beta^{-1} \circ \delta \bar{\rho}\left(l_{1}, l_{2}, l_{3}\right)=\beta^{-1} \circ\left(\bar{\phi}^{\prime}-\bar{\phi}\right)\left(l_{1}, l_{2}, l_{3}\right), \text { for } l_{1}, l_{2}, l_{3} \in L .
$$

Hence $\left(\bar{\phi}^{\prime}-\bar{\phi}\right)=\delta \bar{\rho}$.

Suppose a $B$-bilinear operation $\{$,$\} is given on B \otimes L$, lifting the Leibniz algebra structure [, $]_{\lambda}$ on $A \otimes L$. Then any other $B$-bilinear operation on $B \otimes L$, lifting [, $]_{\lambda}$, is determined by a 2 -cochain $\rho$ as follows. Define $\{,\}^{\prime}:(B \otimes L)^{\otimes 2} \longrightarrow$ $B \otimes L$ by $\left\{l_{1}, l_{2}\right\}^{\prime}=\left\{l_{1}, l_{2}\right\}+I \circ \rho\left(E\left(l_{1}\right), E\left(l_{2}\right)\right)$ for $l_{1}, l_{2} \in B \otimes L$. Then it is easy to see that $\{,\}^{\prime}$ is a lifting of $[,]_{\lambda}$ such that the 2 -cochain $\bar{\rho}$ induced by the difference $\{,\}^{\prime}-\{$,$\} , is the given 2-cochain \rho$.

The above consideration defines a map $\theta_{\lambda}: H_{\text {Harr }}^{2}(A ; \mathbb{K}) \longrightarrow H L^{3}(L ; L)$ by $\theta_{\lambda}([f])=[\bar{\phi}]$, where $[\bar{\phi}]$ is the cohomology class of $\bar{\phi}$. The map $\theta_{\lambda}$ is called the obstruction map.

Proposition 5.6. Let $\lambda$ be a deformation of the Leibniz algebra $L$ with base $A$ and let $B$ be a 1-dimensional extension of $A$ corresponding to the cohomology class $[f] \in H_{\text {Harr }}^{2}(A ; \mathbb{K})$. Then $\lambda$ can be extended to a deformation of $L$ with base $B$ if and only if the obstruction $\theta_{\lambda}([f])=0$.

Proof. Suppose $\theta_{\lambda}([f])=0$. Let

$$
0 \longrightarrow \mathbb{K} \stackrel{i}{\longrightarrow} B \stackrel{p}{\longrightarrow} A \longrightarrow 0
$$

be a 1-dimensional extension representing the cohomology class $[f]$. Let $\{$,$\} be$ a lifting of the Leibniz algebra structure $\lambda$ on $A \otimes L$ to a $B$-bilinear operation on $B \otimes L$. Let $\bar{\phi}$ be the associated cocycle in $C L^{3}(L ; L)$ as described above. Then $\theta_{\lambda}([f])=[\bar{\phi}]=0$ implies $\bar{\phi}=\delta \rho$ for some $\rho \in C L^{2}(L ; L)$. Now take $\rho^{\prime}=-\rho$, and define a new linear map

$$
\{,\}^{\prime}:(B \otimes L)^{\otimes 2} \longrightarrow B \otimes L \text { by }\left\{l_{1}, l_{2}\right\}^{\prime}=\left\{l_{1}, l_{2}\right\}+I \circ \rho^{\prime}\left(E\left(l_{1}\right), E\left(l_{2}\right)\right) .
$$

If $\bar{\phi}^{\prime}$ denotes the cocycle corresponding to $\{,\}^{\prime}$, we have $\bar{\phi}^{\prime}-\bar{\phi}=\delta \overline{\rho^{\prime}}=-\bar{\phi}$. Hence $\bar{\phi}^{\prime}=0$ which implies $\phi^{\prime}=0$. Therefore, $\{,\}^{\prime}$ is a Leibniz algebra structure on $B \otimes L$ extending $\lambda$. The converse is clear.

Assume $\theta_{\lambda}([f])=0$ for $[f] \in H_{\text {Harr }}^{2}(A ; \mathbb{K})$. Let us denote by $S$ the set of all isomorphism classes of deformations $\mu$ of $L$ with base $B$ such that $p_{*} \mu=\lambda$. The group of automorphisms $\mathcal{A}$ of the extension (10) has a natural action $\sigma_{1}$ of $\mathcal{A}$ on $S$, given by $\mu \mapsto u_{*} \mu$ for $u \in \mathcal{A}$. This is clearly well-defined, because if $\mu \cong \mu^{\prime}$, then $u_{*} \mu \cong u_{*} \mu^{\prime}$. 
On the other hand, $\mathbb{H}$ acts on $S$ as follows. Suppose $\mu$ and $\mu^{\prime}$ are two deformations of $L$ with base $B$ such that $p_{*} \mu=p_{*} \mu^{\prime}=\lambda$. Let $\bar{\phi}_{\mu}$ be the 3 -cochain determined by $[,]_{\mu}$ as before. If $\psi \in C L^{2}(L ; L)$ is the cochain determined by $[,]_{\mu}-[,]_{\mu^{\prime}}$, then we have, $\bar{\phi}_{\mu}-\bar{\phi}_{\mu^{\prime}}=\delta \psi$. But $\bar{\phi}_{\mu}=0=\bar{\phi}_{\mu^{\prime}}\left(\mu, \mu^{\prime}\right.$ are the Leibniz brackets). Hence $\psi$ is a cocycle.

Suppose now $\mu_{1} \cong \mu$ with isomorphism $\rho:\left(B \otimes L,[,]_{\mu}\right) \longrightarrow\left(B \otimes L,[,]_{\mu_{1}}\right)$. As explained in the proof of Proposition 4.4 the 2-cochain determined by $[,]_{\mu}-[,]_{\mu_{1}}$ is a coboundary. Hence $[,]_{\mu}-[,]_{\mu_{1}}=\delta b_{\rho}$ for some 1 -cochain $b_{\rho}$, determined by $\rho$. Therefore, $\psi_{1}=[,]_{\mu^{\prime}}-[,]_{\mu_{1}}=\left([,]_{\mu^{\prime}}-[,]_{\mu}\right)+\left([,]_{\mu}-[,]_{\mu_{1}}\right)=\psi+\delta b_{\rho}$. This allows us to introduce a map $\sigma_{2}: \mathbb{H} \times S \longrightarrow S, \sigma_{2}(\psi, \mu)=\mu^{\prime}$, where [, $]_{\mu^{\prime}}-[,]_{\mu}$ determines $\psi$. The above discussion shows that the map is well-defined. It is clear that the action is transitive.

Let us consider the relationship between the two actions $\sigma_{1}$ and $\sigma_{2}$ on $S$.

Proposition 5.7. Let $\lambda$ be a deformation of the Leibniz algebra $L$ with base $A$ and let

$$
0 \longrightarrow \mathbb{K} \stackrel{i}{\longrightarrow} B \stackrel{p}{\longrightarrow} A \longrightarrow 0
$$

be a given extension of $A$. If $u: B \longrightarrow B$ is an automorphism of this extension which corresponds to an element $h \in H_{\text {Harr }}^{1}(A ; \mathbb{K})=T A$, then for any deformation $\mu$ of $L$ with base $B$, such that $p_{*} \mu=\lambda$, the difference $[,]_{u_{*} \mu}-[,]_{\mu}$ is a cocycle in the cohomology class $d \lambda(h)$. This means that the operation $\sigma_{1}$ and $\sigma_{2}$ on $S$ are related to each other by the differential $d \lambda: T A \longrightarrow \mathbb{H}$.

Proof. Recall that $\sigma_{1}: H_{\text {Harr }}^{1}(A ; \mathbb{K}) \times S \longrightarrow S, \sigma_{1}(\phi, \mu)=u_{*} \mu$ where $u$ is the automorphism in $\mathcal{A}$ corresponding to $h=[\phi] \in H_{\text {Harr }}^{1}(A ; \mathbb{K})$, and

$$
\sigma_{2}: \mathbb{H} \times S \longrightarrow S, \sigma_{2}(\psi, \mu)=\mu^{\prime}
$$

where $[,]_{\mu^{\prime}}-[,]_{\mu}$ determines $\psi \in \mathbb{H}$.

We need to show that the 2 -cocycle determined by the difference $[,]_{u_{*} \mu}-[,]_{\mu}$ can be represented by $d \lambda(h)$. One can easily prove this by choosing a basis $\left\{\tilde{m}_{i}\right\}_{1 \leq i \leq l}$ of $\mathfrak{M} / \mathfrak{M}^{2}$ and writing out $[,]_{u_{*} \mu}-[,]_{\mu}$ in terms of $\tilde{m}_{i}$. Namely, using the notations of Section 4 , one gets

$$
[,]_{u_{*} \mu}-[,]_{\mu}=\sum_{i=1}^{l} h\left(\tilde{m}_{i}\right) \otimes \psi_{i}\left(l_{1}, l_{2}\right) .
$$

Thus the cocycle determined by this difference is $\sum_{i=1}^{l} h\left(\tilde{m}_{i}\right) \psi_{i}\left(l_{1}, l_{2}\right)$. On the other hand for the dual basis $\left\{\tilde{\xi}_{i}\right\}_{1 \leq i \leq l}$, if $h=\sum_{i=1}^{l} x_{i} \tilde{\xi}_{i}$ and $x_{i}=h\left(\tilde{m}_{i}\right)$, then $d \lambda(h)=a_{\pi_{*} \lambda}(h)=\sum_{i=1}^{l} h\left(\tilde{m}_{i}\right)\left[\psi_{i}\right]$. This completes the proof. 
Corollory 5.8. Suppose that for a deformation $\lambda$ of the Leibniz algebra $L$ with base $A$, the differential $d \lambda: T A \longrightarrow \mathbb{H}$ is onto. Then the group of automorphisms $\mathcal{A}$ of the extension (10) operates transitively on the set of equivalence classes of deformations $\mu$ of $L$ with base $B$ such that $p_{*} \mu=\lambda$. In other words, if $\mu$ exists, it is unique up to an isomorphism and an automorphism of this extension.

Suppose now that $M$ is a finite dimensional $A$-module satisfying the condition $\mathfrak{M} M=0$, where $\mathfrak{M}$ is the maximal ideal in $A$. The previous results can be generalized from the 1-dimensional extension (10) to a more general extension

$$
0 \longrightarrow M \stackrel{i}{\longrightarrow} B \stackrel{p}{\longrightarrow} A \longrightarrow 0 .
$$

If we try to extend a deformation with base $A$ to a deformation with base $B$, as in the beginning of the this section, then an analogous computation yields

$$
\tilde{\phi}:\left(\frac{B \otimes L}{k e r(E)}\right)^{\otimes 3} \longrightarrow \operatorname{ker}(P)=i m(I) \cong M \otimes L .
$$

It will give rise to $\bar{\phi} \in C L^{3}(L ; M \otimes L)$ with the cohomology class

$$
[\bar{\phi}] \in H L^{3}(L ; M \otimes L)=M \otimes H L^{3}(L ; L) .
$$

The obstruction map for this extension is

$$
\theta_{\lambda}: H_{\text {Harr }}^{2}(A ; M) \longrightarrow M \otimes H L^{3}(L ; L) \text { defined by } \theta_{\lambda}([f])=[\bar{\phi}] .
$$

Then, as in the case of 1-dimensional extension, we have the following.

Proposition 5.9. Let $\lambda$ be a deformation of a Leibniz algebra $L$ with base $(A, \mathfrak{M})$ and let $M$ be a finite dimensional $A$-module with $\mathfrak{M} M=0$. Consider an extension $B$ of $A$

$$
0 \longrightarrow M \stackrel{i}{\longrightarrow} B \stackrel{p}{\longrightarrow} A \longrightarrow 0
$$

corresponding to some $[f] \in H_{\text {Harr }}^{2}(A ; M)$. A deformation $\mu$ of $L$ with base $B$ such that $p_{*} \mu=\lambda$ exists if and only if the obstruction $\theta_{\lambda}([f])=0$. If $d \lambda$ : $T A \longrightarrow \mathbb{H}$ is onto, then the deformation $\mu$, if it exists, is unique up to an isomorphism and an automorphism of the above extension.

We end this section with the following naturality property of the obstruction map.

Proposition 5.10. Suppose $A_{1}$ and $A_{2}$ are finite dimensional local algebras with augmentations $\varepsilon_{1}$ and $\varepsilon_{2}$, respectively. Let $\phi: A_{2} \longrightarrow A_{1}$ be an algebra homomorphism with $\phi(1)=1$ and $\varepsilon_{1} \circ \phi=\varepsilon_{2}$. Suppose $\lambda_{2}$ is a deformation of a Leibniz algebra $L$ with base $A_{2}$ and $\lambda_{1}=\phi_{*} \lambda_{2}$ is the push-out via $\phi$. Then the following diagram commutes. 


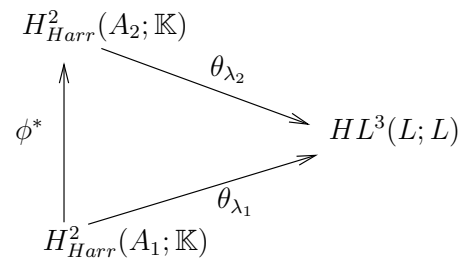

Figure 1:

Proof. Let $\left[f_{A_{1}}\right] \in H_{\text {Harr }}^{2}\left(A_{1} ; \mathbb{K}\right)$ and $\left[f_{A_{2}}\right]=\phi^{*}\left(\left[f_{A_{1}}\right]\right) \in H_{\text {Harr }}^{2}\left(A_{2} ; \mathbb{K}\right)$ correspond to the classes of 1 - dimensional extensions of $A_{1}$ and $A_{2}$, represented by

$$
0 \longrightarrow \mathbb{K} \stackrel{i_{k}}{\longrightarrow} A_{k}^{\prime} \stackrel{p_{k}}{\longrightarrow} A_{k} \longrightarrow 0, \quad k=1,2 .
$$

Fix some sections $q_{k}: A_{k} \longrightarrow A_{k}^{\prime}$ of $p_{k}$ for $k=1,2$. Then, as in (5), we get $\mathbb{K}$-module isomorphisms $A_{k}^{\prime} \cong A_{k} \oplus \mathbb{K}$. Let $(b, x)_{q_{k}}$ denote the inverse of $(b, x)$ under the above isomorphisms. The algebra structures on $A_{k}^{\prime}$ are determined as in (6). Define $\psi: A_{2}^{\prime} \cong\left(A_{2} \oplus \mathbb{K}\right) \longrightarrow A_{1}^{\prime} \cong\left(A_{1} \oplus \mathbb{K}\right)$ by $\psi\left((a, x)_{q_{2}}\right)=(\phi(a), x)_{q_{1}}$ for $(a, x)_{q_{2}} \in A_{2}^{\prime}$. It is clear that $\psi$ is a $\mathbb{K}$-algebra homomorphism. Thus we get a morphism between the two extensions given by

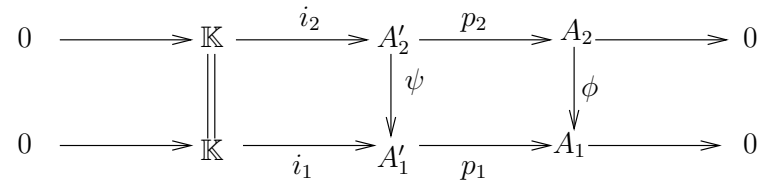

Figure 2:

Let $I_{k}=i_{k} \otimes i d, P_{k}=p_{k} \otimes i d$ and $E_{k}=\hat{\varepsilon}_{k} \otimes i d$, where $\hat{\varepsilon}_{k}=\varepsilon_{k} \circ p_{1}$ for $k=1,2$. Suppose $\mathfrak{M}_{A_{k}}$ is the unique maximal ideal in $A_{k}$. Then $\mathfrak{M}_{A_{k}^{\prime}}=p_{k}^{-1}\left(\mathfrak{M}_{A_{k}}\right)$ is the unique maximal ideal of $A_{k}^{\prime}$. Denote by $\left\{m_{k i}\right\}_{1 \leq i \leq r_{k}}$ a basis of $\mathfrak{M}_{A_{k}}$ and $\left\{n_{k i}\right\}_{1 \leq i \leq r_{k}+1}$ a basis of $\mathfrak{M}_{A_{k}^{\prime}}$ for $k=1,2$. Here $n_{k j}=\left(m_{k j}, 0\right)_{q_{k}}$ for $1 \leq j \leq r_{k}$ and $n_{k\left(r_{k}+1\right)}=(0,1)_{q_{k}}$. By (4), the Leibniz bracket on $A_{2} \otimes L$ is defined by

$$
\left[1 \otimes l_{1}, 1 \otimes l_{2}\right]_{\lambda_{2}}=1 \otimes\left[l_{1}, l_{2}\right]+\sum_{i=1}^{r_{2}} m_{2 i} \otimes \psi_{i}^{2}\left(l_{1}, l_{2}\right) \text { for } l_{1}, l_{2} \in L
$$

and $\psi_{i}^{2}=\alpha_{\lambda_{2} \xi_{2 i}}$, where $\left\{\xi_{2 i}\right\}$ is the dual basis of $\left\{m_{2 i}\right\}$. Let $\phi\left(m_{2 i}\right)=$ $\sum_{j=1}^{r_{1}} c_{i, j} m_{1 j}$ where $c_{i, j} \in \mathbb{K}$ for $1 \leq i \leq r_{2}$ and $1 \leq j \leq r_{1}$. Then the 
push-out $\lambda_{1}=\phi_{*} \lambda_{2}$ on $A_{1} \otimes L$ is defined by

$$
\begin{aligned}
{\left[1 \otimes l_{1}, 1 \otimes l_{2}\right]_{\lambda_{1}} } & =1 \otimes\left[l_{1}, l_{2}\right]+\sum_{i=1}^{r_{2}}\left(\sum_{j=1}^{r_{1}} c_{i, j} m_{1 j}\right) \otimes \psi_{i}^{2}\left(l_{1}, l_{2}\right) \\
& =1 \otimes\left[l_{1}, l_{2}\right]+\sum_{j=1}^{r_{1}} m_{1 j} \otimes \psi_{j}^{1}\left(l_{1}, l_{2}\right) \text { for } l_{1}, l_{2} \in L .
\end{aligned}
$$

Here $\psi_{j}^{1} \in C L^{2}(L ; L)$ is defined by $\psi_{j}^{1}\left(l_{1}, l_{2}\right)=\sum_{i=1}^{r_{2}} c_{i, j} \psi_{i}^{2}\left(l_{1}, l_{2}\right)$ for $l_{1}, l_{2} \in L$. For any 2-cochain $\chi \in C L^{2}(L ; L)$, let $\{,\}_{k}:\left(A_{k}^{\prime} \otimes L\right)^{\otimes 2} \longrightarrow A_{k}^{\prime} \otimes L$ be the $A_{k}^{\prime}$-bilinear operation on $A_{k}^{\prime} \otimes L$ lifting $\lambda_{k}$, defined by

$$
\left\{1 \otimes l_{1}, 1 \otimes l_{2}\right\}_{k}=1 \otimes\left[l_{1}, l_{2}\right]+\sum_{j=1}^{r_{k}} n_{k j} \otimes \psi_{j}^{k}\left(l_{1}, l_{2}\right)+n_{k\left(r_{k}+1\right)} \chi\left(l_{1}, l_{2}\right)
$$

for $k=1,2$ and $l_{1}, l_{2} \in L$. We know that $\{,\}_{k}$ satisfies properties $(i)$ and (ii) of (7).

We claim that $\psi \otimes i d$ preserves the liftings. It is enough to show that $(\psi \otimes i d)\left(\left\{1 \otimes l_{1}, 1 \otimes l_{2}\right\}_{2}\right)=\left\{\psi \otimes i d\left(1 \otimes l_{1}\right), \psi \otimes i d\left(1 \otimes l_{2}\right)\right\}_{1}$ for $l_{1}, l_{2} \in L$. Now

$(\psi \otimes i d)\left(\left\{1 \otimes l_{1}, 1 \otimes l_{2}\right\}_{2}\right)$

$=\psi(1) \otimes\left[l_{1}, l_{2}\right]+\sum_{j=1}^{r_{2}} \psi(1) \psi\left(n_{2 j}\right) \otimes \psi_{j}^{2}\left(l_{1}, l_{2}\right)+\psi(1) \psi\left(n_{2\left(r_{2}+1\right)}\right) \otimes \chi\left(l_{1}, l_{2}\right)$

$=1 \otimes\left[l_{1}, l_{2}\right]+\sum_{j=1}^{r_{2}}\left(\sum_{i=1}^{r_{1}} c_{j, i} m_{1 i}\right) \otimes \psi_{j}^{2}\left(l_{1}, l_{2}\right)+n_{1\left(r_{1}+1\right)} \otimes \chi\left(l_{1}, l_{2}\right)$

$\left(\phi\left(m_{2 j}\right)=\sum_{i=1}^{r_{1}} c_{j, i} m_{1 i}\right.$ and $\left.\psi\left(n_{2\left(r_{2}+1\right)}\right)=\psi\left((0,1)_{q_{2}}\right)=(\phi(0), 1)_{q_{1}}=n_{1\left(r_{1}+1\right)}\right)$

$=\psi(1) \otimes\left[l_{1}, l_{2}\right]+\sum_{i=1}^{r_{1}} \psi(1) m_{1 i} \otimes \psi_{i}^{1}\left(l_{1}, l_{2}\right)+\psi(1) n_{1\left(r_{1}+1\right)} \otimes \chi\left(l_{1}, l_{2}\right)$

$=\left\{\psi(1) \otimes l_{1}, \psi(1) \otimes l_{2}\right\}_{1}$

$=\left\{\psi \otimes i d\left(1 \otimes l_{1}\right), \psi \otimes i d\left(1 \otimes l_{2}\right)\right\}_{1}, \quad$ which proves our claim.

Let $\phi_{k}$ be defined by $\{,\}_{k}$ as in (8) and $\bar{\phi}_{k}$ the corresponding cocycle as in (9). As $\psi\left(n_{2\left(r_{2}+1\right)}\right)=n_{1\left(r_{1}+1\right)}$, it follows from the definition of $\phi_{k}$ and the previous claim that $\left[\overline{\phi_{2}}\right]=\left[\overline{\phi_{1}}\right]$. Therefore, $\theta_{\lambda_{1}}\left(\left[f_{A_{1}}\right]\right)=\left[\overline{\phi_{1}}\right]=\left[\overline{\phi_{2}}\right]=\theta_{\lambda_{2}}\left(\left[f_{A_{2}}\right]\right)=$ $\theta_{\lambda_{2}} \circ \phi^{*}\left(\left[f_{A_{1}}\right]\right)$. Hence $\theta_{\lambda_{1}}=\theta_{\lambda_{2}} \circ \phi^{*}$.

\section{Construction of a Versal Deformation}

In this section we give an explicit construction of versal deformation of a given Leibniz algebra following [5]. 
Consider the Leibniz algebra $L$ with $\operatorname{dim}(\mathbb{H})<\infty$. Set $C_{0}=\mathbb{K}$ and $C_{1}=$ $\mathbb{K} \oplus \mathbb{H}^{\prime}$. Consider the extension

$$
0 \longrightarrow \mathbb{H}^{\prime} \stackrel{i}{\longrightarrow} C_{1} \stackrel{p}{\longrightarrow} C_{0} \longrightarrow 0,
$$

where the multiplication in $C_{1}$ is defined by

$$
\left(k_{1}, h_{1}\right) \cdot\left(k_{2}, h_{2}\right)=\left(k_{1} k_{2}, k_{1} h_{2}+k_{2} h_{1}\right) \text { for }\left(k_{1}, h_{1}\right),\left(k_{2}, h_{2}\right) \in C_{1} .
$$

Let $\eta_{1}$ be the universal infinitesimal deformation with base $C_{1}$ as constructed in Section 4 . We proceed by induction. Suppose for some $k \geq 1$ we have constructed a finite dimensional local algebra $C_{k}$ and a deformation $\eta_{k}$ of $L$ with base $C_{k}$. Let

$$
\mu: H_{\text {Harr }}^{2}\left(C_{k} ; \mathbb{K}\right) \longrightarrow\left(C h_{2}\left(C_{k}\right)\right)^{\prime}
$$

be a homomorphism sending a cohomology class to a cocycle representing the class. Let

$$
f_{C_{k}}: C h_{2}\left(C_{k}\right) \longrightarrow H_{\text {Harr }}^{2}\left(C_{k} ; \mathbb{K}\right)^{\prime}
$$

be the dual of $\mu$. By Proposition 5.4 (ii) we have the following extension of $C_{k}$ :

$$
0 \longrightarrow H_{\text {Harr }}^{2}\left(C_{k} ; \mathbb{K}\right)^{\prime} \stackrel{\bar{i}_{k+1}}{\longrightarrow} \bar{C}_{k+1} \stackrel{\bar{p}_{k+1}}{\longrightarrow} C_{k} \longrightarrow 0 .
$$

The corresponding obstruction $\theta\left(\left[f_{C_{k}}\right]\right) \in H_{H a r r}^{2}\left(C_{k} ; \mathbb{K}\right)^{\prime} \otimes H L^{3}(L ; L)$ gives a linear map $\omega_{k}: H_{H a r r}^{2}\left(C_{k} ; \mathbb{K}\right) \longrightarrow H L^{3}(L ; L)$ with the dual map

$$
\omega_{k}{ }^{\prime}: H L^{3}(L ; L)^{\prime} \longrightarrow H_{\text {Harr }}^{2}\left(C_{k} ; \mathbb{K}\right)^{\prime} .
$$

We have an induced extension

$$
0 \longrightarrow \operatorname{coker}\left(\omega_{k}^{\prime}\right) \longrightarrow \bar{C}_{k+1} / \bar{i}_{k+1} \circ \omega_{k}^{\prime}\left(H L^{3}(L ; L)^{\prime}\right) \longrightarrow C_{k} \longrightarrow 0 .
$$

Since $\operatorname{coker}\left(\omega_{k}^{\prime}\right) \cong\left(\operatorname{ker}\left(\omega_{k}\right)\right)^{\prime}$, it yields an extension

$$
0 \longrightarrow\left(k e r\left(\omega_{k}\right)\right)^{\prime} \stackrel{i_{k+1}}{\longrightarrow} C_{k+1} \stackrel{p_{k+1}}{\longrightarrow} C_{k} \longrightarrow 0
$$

where $C_{k+1}=\bar{C}_{k+1} / \bar{i}_{k+1} \circ \omega_{k}^{\prime}\left(H L^{3}(L ; L)^{\prime}\right)$ and $i_{k+1}, p_{k+1}$ are the mappings induced by $\bar{i}_{k+1}$ and $\bar{p}_{k+1}$, respectively. Observe that the algebra $C_{k}$ is also local. Since $C_{k}$ is finite dimensional, the cohomology group $H_{H a r r}^{2}\left(C_{k} ; \mathbb{K}\right)$ is also finite dimensional and hence $C_{k+1}$ is finite dimensional as well.

Remark 6.1. It follows from Proposition 5.2 that the specific extension (11) has the following "universality property". For any $C_{k}$-module $M$ with $\mathfrak{M} M=0$, (11) admits a unique morphism into an arbitrary extension of $C_{k}$ :

$$
0 \longrightarrow M \longrightarrow B \longrightarrow C_{k} \longrightarrow 0 \text {. }
$$


Proposition 6.2. The deformation $\eta_{k}$ with base $C_{k}$ of a Leibniz algebra $L$ admits an extension to a deformation with base $C_{k+1}$, which is unique up to an isomorphism and an automorphism of the extension

$$
0 \longrightarrow\left(k e r\left(\omega_{k}\right)\right)^{\prime} \stackrel{i_{k+1}}{\longrightarrow} C_{k+1} \stackrel{p_{k+1}}{\longrightarrow} C_{k} \longrightarrow 0 .
$$

Proof. From the above construction of the extension (12) it is clear that the corresponding obstruction map is the restriction of $\omega_{k}$,

$$
\left.\omega_{k}\right|_{\operatorname{ker}\left(\omega_{k}\right)}: \operatorname{ker}\left(\omega_{k}\right) \longrightarrow H L^{3}(L ; L) .
$$

Hence, it is the zero map. Thus the result follows from Proposition 5.9

By induction, the above process yields a sequence of finite dimensional local algebras $C_{k}$ and deformations $\eta_{k}$ of the Leibniz algebra $L$ with base $C_{k}$

$$
\mathbb{K} \stackrel{p_{1}}{\longleftarrow} C_{1} \stackrel{p_{2}}{\longleftarrow} C_{2} \stackrel{p_{3}}{\longleftarrow} \ldots \ldots \stackrel{p_{k}}{\longleftarrow} C_{k} \stackrel{p_{k+1}}{\longleftarrow} C_{k+1} \ldots
$$

such that $p_{k+1} \eta_{k+1}=\eta_{k}$. Thus by taking the projective limit we obtain a formal deformation $\eta$ of $L$ with base $C=\lim _{k \rightarrow \infty} C_{k}$.

Next, we give an algebraic description of the base $C$ of the versal deformation. For that we need the following Proposition from [12].

Proposition 6.3. Let $A=\mathbb{K}\left[x_{1}, x_{2}, \ldots, x_{n}\right]$ be the polynomial algebra, and let $\mathfrak{M}$ be the ideal of polynomials without constant terms.

(a) If an ideal I of $A$ is contained in $\mathfrak{M}^{2}$, then $H_{\text {Harr }}^{2}(A / I ; \mathbb{K}) \cong(I / \mathfrak{M} I)^{\prime}$.

(b) There is an extension for $B=A / I$ :

$$
0 \longrightarrow I / \mathfrak{M} I \stackrel{i}{\longrightarrow} A / \mathfrak{M} I \stackrel{p}{\longrightarrow} A / I \longrightarrow 0
$$

where $i$ and $p$ are induced by the inclusions $I \hookrightarrow A$ and $\mathfrak{M} I \hookrightarrow I$.

Suppose $\operatorname{dim}(\mathbb{H})=n$. Let $\left\{h_{i}\right\}_{1 \leq i \leq n}$ be a basis of $\mathbb{H}$ and $\left\{g_{i}\right\}_{1 \leq i \leq n}$ be the corresponding dual basis. Let $\mathbb{K}\left[\left[\mathbb{H}^{\prime}\right]\right]$ denote the formal power series ring $\mathbb{K}\left[\left[g_{1}, \ldots, g_{n}\right]\right]$ in $n$ variables $g_{1}, \ldots, g_{n}$ over $\mathbb{K}$. Now a typical element in $\mathbb{K}\left[\left[\mathbb{H}^{\prime}\right]\right]$ is of the form

$$
\sum_{i=0}^{\infty} a_{i} f_{i}\left(g_{1}, \ldots, g_{n}\right)=a_{0}+a_{1} f_{1}\left(g_{1}, \ldots, g_{n}\right)+a_{2} f_{2}\left(g_{1}, \ldots, g_{n}\right)+\ldots
$$

where $a_{i} \in \mathbb{K}$ and $f_{i}$ is a monomial of degree $i$ in $n$-variables $g_{1}, \ldots, g_{n}$ for $i=0,1,2, \ldots$ Let $\mathfrak{M}$ denote the unique maximal ideal in $\mathbb{K}\left[\left[\mathbb{H}^{\prime}\right]\right]$, consisting of all elements in $\mathbb{K}\left[\left[\mathbb{H}^{\prime}\right]\right]$ with constant term being equal to zero. 
Proposition 6.4. For the local algebra $C_{k}$ we have $C_{k} \cong \mathbb{K}\left[\left[\mathbb{H}^{\prime}\right]\right] / I_{k}$ for some ideal $I_{k}$, satisfying $\mathfrak{M}^{2}=I_{1} \supset I_{2} \supset \ldots \supset I_{k} \supset \mathfrak{M}^{k+1}$.

Proof. By construction, $C_{1}=\mathbb{K} \oplus \mathbb{H}^{\prime} \cong \mathbb{K}\left[\left[\mathbb{H}^{\prime}\right]\right] / \mathfrak{M}^{2}$. Suppose we already know that $C_{k} \cong \mathbb{K}\left[\left[\mathbb{H}^{\prime}\right]\right] / I_{k}$ where $\mathfrak{M}^{2} \supset I_{k} \supset \mathfrak{M}^{k+1}$. Then by specifying $A=\mathbb{K}\left[\left[\mathbb{H}^{\prime}\right]\right]$ and $I=I_{k}$ in Proposition 6.3. we get $\bar{C}_{k+1} \cong \mathbb{K}\left[\left[\mathbb{H}^{\prime}\right]\right] / \mathfrak{M} I_{k}$. In the previous construction, $C_{k+1}$ is the quotient of $\bar{C}_{k+1}$ by an ideal contained in $I_{k} / \mathfrak{M} I_{k} \subset$ $\mathfrak{M}^{2} / \mathfrak{M} I_{k}$. Hence $C_{k+1} \cong \mathbb{K}\left[\left[\mathbb{H}^{\prime}\right]\right] / I_{k+1}$ where $\mathfrak{M}^{2} \supset I_{k+1} \supset \mathfrak{M} I_{k} \supset \mathfrak{M}^{k+2}$. The proof is now complete by induction.

Corollory 6.5. For $k \geq 2$ the projection $p_{k}: C_{k} \longrightarrow C_{k-1}$ induces an isomorphism $T C_{k} \longrightarrow T C_{k-1}$. In particular, for every $k \geq 1, T C_{k} \cong T C_{1}=\mathbb{H}$. Moreover, under the above identification of $T C_{k}$ with $\mathbb{H}$, the differential $d \eta_{k}$ : $T C_{k} \longrightarrow \mathbb{H}$ is the identity map.

Proof. We have $C_{0}=\mathbb{K} ; C_{1}=\mathbb{K} \oplus \mathbb{H}^{\prime} \cong \mathbb{K}\left[\left[\mathbb{H}^{\prime}\right]\right] / \mathfrak{M}^{2}$ and for $k \geq 2, C_{k}=$ $\mathbb{K}\left[\left[\mathbb{H}^{\prime}\right]\right] / I_{k}$ where $\mathfrak{M}^{2}=I_{1} \supset I_{2} \supset \ldots \supset I_{k} \supset \mathfrak{M}^{k+1}$. The projection $p_{k}: C_{k} \longrightarrow$ $C_{k-1}$ is given by $p_{k}\left(f+I_{k}\right)=f+I_{k-1}$ for $f \in C_{k}$ and $k \geq 1$. The map $p_{k}$ gives rise to a surjective linear map $\mathfrak{M} / I_{k} \longrightarrow \mathfrak{M} / I_{k-1}$. Taking the quotient map $\mathfrak{M} / I_{k-1} \longrightarrow \frac{\mathfrak{M} / I_{k-1}}{\mathfrak{M}^{2} / I_{k-1}}$, we get an epimorphism $\mathfrak{M} / I_{k} \longrightarrow \frac{\mathfrak{M} / I_{k-1}}{\mathfrak{M}^{2} / I_{k-1}}$ with kernel $\mathfrak{M}^{2} / I_{k}$ which corresponds to an isomorphism

$$
\frac{\mathfrak{M} / I_{k}}{\mathfrak{M}^{2} / I_{k}} \longrightarrow \frac{\mathfrak{M} / I_{k-1}}{\mathfrak{M}^{2} / I_{k-1}}
$$

As a result we get an isomorphism

$$
\left(\frac{\mathfrak{M} / I_{k}}{\mathfrak{M}^{2} / I_{k}}\right)^{\prime}=T C_{k} \longrightarrow T C_{k-1}=\left(\frac{\mathfrak{M} / I_{k-1}}{\mathfrak{M}^{2} / I_{k-1}}\right)^{\prime} .
$$

Observe that for any $k \geq 1, T C_{k}=\left(\frac{\mathfrak{M} / I_{k}}{\mathfrak{M}^{2} / I_{k}}\right)^{\prime}=\left(\frac{\mathfrak{M}}{\mathfrak{M} 2}\right)^{\prime} \cong T C_{1}$. On the other hand, since $C_{1}=\mathbb{K} \oplus \mathbb{H}^{\prime}$ with maximal ideal $\mathbb{H}^{\prime}$ and $\left(\mathbb{H}^{\prime}\right)^{2}=0$. Hence $T C_{1}=$ $\left(\mathbb{H}^{\prime}\right)^{\prime}=\mathbb{H}$. The last assertion follows from the definition of the differential.

Proposition 6.6. The complete local algebra $C=\lim _{k \rightarrow \infty} C_{k}$ can be described as $C \cong \mathbb{K}\left[\left[\mathbb{H}^{\prime}\right]\right] / I$, where $I$ is an ideal contained in $\mathfrak{M}^{2}$.

Proof. Consider the map

$$
\phi: \mathbb{K}\left[\left[\mathbb{H}^{\prime}\right]\right] \longrightarrow C_{k}=\mathbb{K}\left[\left[\mathbb{H}^{\prime}\right]\right] / I_{k} \text { defined by } \phi(f)=f+I_{k} \text { for } f \in \mathbb{K}\left[\left[\mathbb{H}^{\prime}\right]\right] \text {. }
$$

Since $I_{k} \supset \mathfrak{M}^{k+1}$, the map $\phi$ induces an epimorphism

$$
\phi_{k}: \mathbb{K}\left[\left[\mathbb{H}^{\prime}\right]\right] / \mathfrak{M}^{k+1} \longrightarrow C_{k} \text { for each } k \geq 1 .
$$


In the limit we get an epimorphism

$$
\mathbb{K}\left[\left[\mathbb{H}^{\prime}\right]\right]=\lim _{k \rightarrow \infty} \mathbb{K}\left[\left[\mathbb{H}^{\prime}\right]\right] / \mathfrak{M}^{k+1} \longrightarrow \lim _{k \rightarrow \infty} C_{k} .
$$

Therefore $C \cong \mathbb{K}\left[\left[\mathbb{H}^{\prime}\right]\right] / I$ where $I=\bigcap_{k} I_{k}$ is the kernel of the epimorphism.

Finally we prove the versality property of the constructed deformation $\eta$ with base $C$. For this we use the following standard lemma.

Lemma 6.7. Suppose $0 \longrightarrow M_{s} \stackrel{i}{\longrightarrow} B_{s} \stackrel{p}{\longrightarrow} A \longrightarrow 0$ is an s-dimensional extension of $A$. Then there exists an $(s-1)$-dimensional extension

$$
0 \longrightarrow M_{s-1} \stackrel{\bar{i}}{\longrightarrow} B_{s-1} \stackrel{\bar{p}}{\longrightarrow} A \longrightarrow 0
$$

of $A$ and a 1-dimensional extension

$$
0 \longrightarrow \mathbb{K} \stackrel{i^{\prime}}{\longrightarrow} B_{s} \stackrel{p^{\prime}}{\longrightarrow} B_{s-1} \longrightarrow 0 .
$$

Theorem 6.8. Let $L$ be a Leibniz algebra with $\operatorname{dim}(\mathbb{H})<\infty$. Then the formal deformation $\eta$ with base $C$ constructed above is a versal deformation of $L$.

Proof. Suppose $\operatorname{dim}(\mathbb{H})=n$. Let $\left\{h_{i}\right\}_{1 \leq i \leq n}$ be a basis of $\mathbb{H}$ and $\left\{g_{i}\right\}_{1 \leq i \leq n}$ the corresponding dual basis of $\mathbb{H}^{\prime}$. Let $A$ be a complete local algebra with maximal ideal $\mathfrak{M}$ and let $\lambda$ be a formal deformation of $L$ with base $A$. We want to find a $\mathbb{K}$-algebra homomorphism $\phi: C \longrightarrow A$ such that $\phi_{*} \eta=\lambda$. Denote $A_{0}=A / \mathfrak{M} \cong \mathbb{K} ; A_{1}=A / \mathfrak{M}^{2} \cong \mathbb{K} \oplus(T A)^{\prime}$. Since $A$ is complete, we have $A=\lim _{k \rightarrow \infty} A / \mathfrak{M}^{k}$. Moreover, for each $k$ we have the following finite dimensional extension

$$
0 \longrightarrow \frac{\mathfrak{M}^{k}}{\mathfrak{M}^{k+1}} \longrightarrow \frac{A}{\mathfrak{M}^{k+1}} \longrightarrow \frac{A}{\mathfrak{M}^{k}} \longrightarrow 0,
$$

because $\operatorname{dim}\left(\frac{\mathfrak{M}^{k}}{\mathfrak{M}^{k+1}}\right)<\infty$.

Let $\operatorname{dim}\left(\frac{\mathfrak{M}^{k}}{\mathfrak{M}^{k+1}}\right)=n_{k-1}$. A repeated application of Lemma 6.7 to the extension

$$
0 \longrightarrow \frac{\mathfrak{M}^{2}}{\mathfrak{M}^{3}} \longrightarrow \frac{A}{\mathfrak{M}^{3}} \longrightarrow \frac{A}{\mathfrak{M}^{2}}=A_{1} \longrightarrow 0
$$

yields $n_{1}$ number of 1-dimensional extensions as follows.

$$
\begin{gathered}
0 \longrightarrow \mathbb{K} \longrightarrow A_{2} \longrightarrow A_{1} \longrightarrow 0 \\
0 \longrightarrow \mathbb{K} \longrightarrow A_{3} \longrightarrow A_{2} \longrightarrow 0 \\
\vdots \\
0 \longrightarrow \mathbb{K} \longrightarrow A_{n_{1}+1}=\frac{A}{\mathfrak{M}^{3}} \longrightarrow A_{n_{1}} \longrightarrow 0 .
\end{gathered}
$$


Similarly, the extension

$$
0 \longrightarrow \frac{\mathfrak{M}^{3}}{\mathfrak{M}^{4}} \longrightarrow \frac{A}{\mathfrak{M}^{4}} \longrightarrow \frac{A}{\mathfrak{M}^{3}}=A_{n_{1}+1} \longrightarrow 0
$$

splits into $n_{2}$ number of 1-dimensional extensions and so on. Thus we get a sequence of 1- dimensional extensions

$$
0 \longrightarrow \mathbb{K} \stackrel{j_{k+1}}{\longrightarrow} A_{k+1} \stackrel{q_{k+1}}{\longrightarrow} A_{k} \longrightarrow 0 \quad ; k \geq 1 .
$$

Since $A=\lim _{k \rightarrow \infty} A / \mathfrak{M}^{k}$, it is clear that $A=\lim _{k \rightarrow \infty} A_{k}$. Let $Q_{k}: A \longrightarrow A_{k}$ be the projection map for the inverse system $\left\{A_{k}, q_{k}\right\}_{k \geq 1}$ with the limit $A$, where $Q_{1}: A \longrightarrow A_{1}=A / \mathfrak{M}^{2}$ is the natural projection. Let $Q_{k_{*}} \lambda=\lambda_{k}$, then $\lambda_{k}$ is a deformation of $L$ with base $A_{k}$. Thus $\lambda_{k}=Q_{k_{*}} \lambda=\left(q_{k+1} \circ Q_{k+1}\right)_{*} \lambda=$ $q_{k+1_{*}} \lambda_{k+1}$. Now we will construct inductively homomorphisms $\phi_{j}: C_{j} \longrightarrow A_{j}$ for $j=1,2 \ldots$, compatible with the corresponding projections $C_{j+1} \longrightarrow C_{j}$ and $A_{j+1} \longrightarrow A_{j}$, along with the conditions $\phi_{j *} \eta_{j} \cong \lambda_{j}$. Define

$$
\phi_{1}: C_{1} \longrightarrow A_{1} \quad \text { as } \quad i d \oplus(d \lambda)^{\prime}: \mathbb{K} \oplus \mathbb{H}^{\prime} \longrightarrow \mathbb{K} \oplus(T A)^{\prime} .
$$

From Proposition 4.4 we have $\phi_{1 *} \eta_{1} \cong \lambda_{1}$.

Suppose we have constructed a $\mathbb{K}$-algebra homomorphism $\phi_{k}: C_{k} \longrightarrow$ $A_{k}$ with $\phi_{k *} \eta_{k} \cong \lambda_{k}$. Consider the homomorphism $\phi_{k}^{*}: H_{H a r r}^{2}\left(A_{k} ; \mathbb{K}\right) \longrightarrow$ $H_{\text {Harr }}^{2}\left(C_{k} ; \mathbb{K}\right)$ induced by $\phi_{k}$. Let

$$
0 \longrightarrow \mathbb{K} \stackrel{i_{k+1}}{\longrightarrow} B \stackrel{p_{k+1}}{\longrightarrow} C_{k} \longrightarrow 0
$$

represent the image under $\phi_{k}^{*}$ of the isomorphism class of extension

$$
0 \longrightarrow \mathbb{K} \stackrel{j_{k+1}}{\longrightarrow} A_{k+1} \stackrel{q_{k+1}}{\longrightarrow} A_{k} \longrightarrow 0
$$

(see Proposition 5.4). Then we have the following commutative diagram

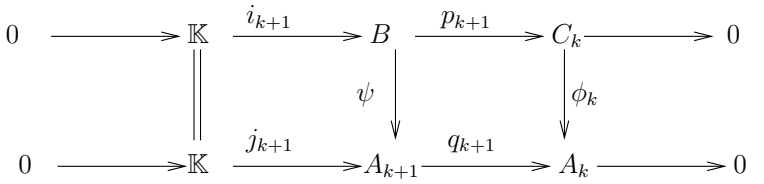

Figure 3:

where $\psi$ is given by $\psi\left((x, k)_{q}\right)=\left(\phi_{k}(x), k\right)_{q^{\prime}}$ for some fixed sections $q$ and $q^{\prime}$ of $p_{k+1}$ and $q_{k+1}$ respectively. Observe that by Proposition 5.10 the obstructions 
in extending $\lambda_{k}$ to the base $A_{k+1}$ and that of $\eta_{k}$ to the base $B$ coincide. Since $\lambda_{k}$ has an extension $\lambda_{k+1}$, the corresponding obstruction is zero. Hence there exists a deformation $\xi$ of $L$ with base $B$ which extends $\eta_{k}$ with base $C_{k}$ such that $\psi_{*} \xi=\lambda_{k+1}$. By Remark 6.1 we get the following unique morphism of extensions.

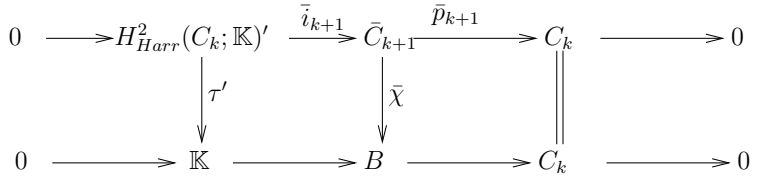

Figure 4:

Since the deformation $\eta_{k}$ has been extended to $B$, the obstruction map

$$
\omega_{k}: H_{H a r r}^{2}\left(C_{k} ; \mathbb{K}\right) \longrightarrow H L^{3}(L ; L)
$$

is zero. Therefore the composition $\tau^{\prime} \circ \omega_{k}^{\prime}: H L^{3}(L ; L)^{\prime} \longrightarrow \mathbb{K}$ is zero. So $\tau^{\prime}$ will induce a linear map $\tau: H_{H a r r}^{2}\left(C_{k} ; \mathbb{K}\right)^{\prime} / \omega_{k}^{\prime}\left(H L^{3}(L ; L)^{\prime}\right) \longrightarrow \mathbb{K}$. Also the map $\bar{\chi}: \bar{C}_{k+1} \longrightarrow B$ will induce a linear map $\chi: C_{k+1}=\bar{C}_{k+1} / \bar{i}_{k+1} \circ$ $\omega_{k}^{\prime}\left(H L^{3}(L ; L)^{\prime}\right) \longrightarrow B$. Since coker $\left(\omega_{k}^{\prime}\right) \cong\left(k e r\left(\omega_{k}\right)\right)^{\prime}$, the last diagram yields the following commutative diagram.

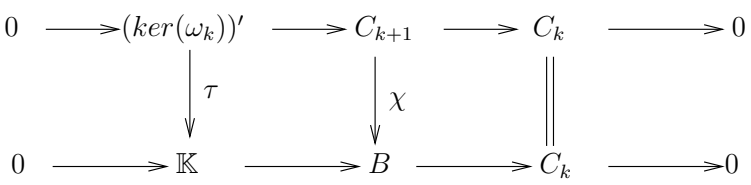

Figure 5:

By Corollary 6.5, the differential

$$
d \eta_{k}: T C_{k} \longrightarrow \mathbb{H}
$$

is onto, so by Corollary 5.8 , the deformations $\chi_{*} \eta_{k+1}$ and $\xi$ are related by some automorphism $u: B \longrightarrow B$ of the extension

$$
0 \longrightarrow \mathbb{K} \longrightarrow B \longrightarrow C_{k} \longrightarrow 0
$$

with $u_{*}\left(\chi_{*} \eta_{k+1}\right)=\xi$. Now set $\phi_{k+1}=(\psi \circ u \circ \chi): C_{k+1} \longrightarrow A_{k+1}$, where $\psi$ is as in Figure 3 . Then

$$
\phi_{k+1_{*}} \eta_{k+1}=\psi_{*} \circ u_{*} \circ \chi_{*} \eta_{k+1}=\psi_{*} \xi=\lambda_{k+1} .
$$


Thus by induction we get a sequence of homomorphisms $\phi_{k}: C_{k} \longrightarrow A_{k}$ with $\phi_{k_{*}} \eta_{k}=\lambda_{k}$. Consequently, taking the limit, we find a homomorphism $\phi: C \longrightarrow$ $A$ such that $\phi_{*} \eta=\lambda$. If $\mathfrak{M}^{2}=0$, then the uniqueness of $\phi$ follows from the corresponding property in Proposition 4.4.

\section{Conclusions}

In this work we gave a constructive method for Leibniz algebras for the solution of the main deformation question, suitable for specific computations. The main feature of this method is that it completely describes all non-equivalent deformations - a problem which did not have a satisfactory solution for a long time. For this we had to consider deformations with complete local algebra base, which was necessary for the existence of a versal deformation. The construction presented here is an inductive procedure, which consists of extending the base of deformation at each step. The specific description of the base of the versal deformation is useful for computations.

\section{References}

[1] S. Albeverio, Sh. A. Ayupov and B.A. Omirov, On nilpotent and simple Leibniz algebras, Comm. Alg., 33 (2005), 159-172.

[2] S. Albeverio, B.A. Omirov and I.S. Rakhimov, Varieties of nilpotent complex Leibniz algebras of dimension less than five, Comm. Alg., 33(2005), 15751585 .

[3] D. Balavoine, Deformation of algebras over a quadratic operads, Contemporary Maths. AMS, 202 (1997) 207-234.

[4] A. Fialowski, An example of formal deformations of Lie algebras, "NATO Conference on deformation theory of algebras and applications, Proceedings", Kluwer, Dordrecht, (1988), 375-401.

[5] A. Fialowski and D. Fuchs Construction of versal Deformation of Lie Algebras, Journal of Functional Analysis 161 (1999),76-110.

[6] M. Gerstenhaber, On the Deformations of Rings and Algebras, Ann. of Math. 79 (1964), 59-103.

[7] M. Gerstenhaber, On the Deformations of Rings and Algebras, Ann. of Math. 84 (1966), 1-19. 
[8] M. Gerstenhaber On the Deformations of Rings and Algebras, Ann. of Math. 88 (1968), 1-34.

[9] M. Gerstenhaber, On the Deformations of Rings and Algebras, Ann. of Math. 99 (1974), 257-276.

[10] M. Gerstenhaber, The Cohomology Structure of an Associative Ring, Ann. of Math. 78, (1963), 267-288.

[11] W.M. Goldman and J.J. Millson, The deformation theory of representations of fundamental groups of compact Kähler manifolds, Inst. Hautes Etudes Sci. Pub. Math. 67 (1988), 43-96.

[12] D. K Harrison, Commutative algebras and cohomology, Trans. Amer. Math. Soc. 104 (1962), 191-204.

[13] R. Hartshorne, "Algebraic Geometry", Springer-Verlag, Berlin/New York, 1977.

[14] L. Illusie, "Complexe cotangent et deformations I", Lecture Notes in Math., 239, Springer- Verlag, Berlin/New York, 1971.

[15] M. Kontsevich, "Topics in Algebra: Deformation Theory", Lecture Notes, Univ. of California Press, Berkeley, CA, 1994.

[16] O.A. Laudal, "Formal moduli of algebraic structures", Lecture Notes in Math.,754, Springer-Verlag, Berlin/New York, 1979.

[17] Loday J.-L, Une version con-commutative des algebres de Lie: Les algebres de Leibniz, Enseign. Math., 39, No.3-4 (1993), 269-293.

[18] Loday J.-L, Overview on Leibniz algebras, dialgebras and their homology, Fields Institute Communications, 17 (1997), 91-102.

[19] Loday J.-L, Dialgebras and related operads, Lecture Notes in Math, 1763, 7-66, 2001.

[20] Loday J.-L and Pirashvili Universal enveloping algebras of Leibniz algebras and (co)homology, Math.Ann., 296 (1993),139-158.

[21] V.P. Palamodov, Deformations of complex spaces, Russian Math. Surveys 31 (1976).

[22] M. Schlessinger, Functors of Artin rings, Trans. Amer. Math. Soc. 130 (1968), 208-222. 


\section{Alice Fialowski}

Eötvös Loránd University, Budapest, Hungary.

e-mail: fialowsk@cs.elte.hu

\section{Ashis Mandal}

Indian Statistical Institute, Kolkata, India.

e-mail: ashis_r@isical.ac.in

\section{Goutam Mukherjee}

Indian Statistical Institute, Kolkata, India.

e-mail: goutam@isical.ac.in 